Hande Yaman

\title{
Formulations and Valid Inequalities for the Heterogeneous Vehicle Routing Problem
}

Received: November 17, 2003 / Accepted: May 30, 2005

Published online: July 14, 2005 - @ C Springer-Verlag 2005

\begin{abstract}
We consider the vehicle routing problem where one can choose among vehicles with different costs and capacities to serve the trips. We develop six different formulations: the first four based on MillerTucker-Zemlin constraints and the last two based on flows. We compare the linear programming bounds of these formulations. We derive valid inequalities and lift some of the constraints to improve the lower bounds. We generalize and strengthen subtour elimination and generalized large multistar inequalities.
\end{abstract}

Key words. Heterogeneous vehicle routing problem-Mix fleet-Valid inequalities-Lifting-projection

\section{Introduction}

The heterogeneous (also called mix fleet) vehicle routing problem $(H V R P)$ is defined as follows. We are given a set of nodes where one node is specified as the origin. Each node other than the origin has a fixed demand. A set of vehicle types is given. The aim is to find least cost trips that start and terminate at the origin and choose a vehicle type to serve each trip so that each node other than the origin is served by a trip and the total demand on any trip does not exceed the capacity of the vehicle. The cost of a trip is a linear function of the fixed cost for the vehicle and the distance traveled on the trip.

The $H V R P$ generalizes the classical capacitated vehicle routing problem $(C V R P)$ by considering different types of vehicles. Vehicles may differ in capacity, fixed and variable costs, speed, maximum travel time, and availability. In this paper, we assume that vehicles are different only in capacity and fixed costs.

Despite the huge literature on optimization methods for the $C V R P$ (see e.g. [8], [18], [19], [31], and [32]), the $H V R P$ has received much less attention from the Operations Research community. The research on this problem is focused on heuristic methods and we are not aware of any exact method. The only study about lower bounds is by Golden et al. [12].

The reason for the lack of interest for finding good lower bounds and exact algorithms for the $H V R P$ may be the difficulty of the problem. The $C V R P$ is a special case of the $H V R P$ with one type of vehicle. Another special case where routing costs are zero can be modeled as a binpacking problem with different types of bins and with the objective of minimizing the cost of bins used. If the type of vehicle that traverses

H. Yaman: Department of Industrial Engineering, Bilkent University, Bilkent, 06800 Ankara, Turkey. e-mail: hyaman@bilkent. edu.tr 
each node is given, then the $H V R P$ decomposes into a series of $C V R P$ 's, one for each vehicle type.

A formulation of the $H V R P$ is given in Golden et al. [12] but no computation is reported. The authors derive lower bounds through a combinatorial relaxation. They also present several heuristics and give percentage gaps computed using the best upper bound and the lower bound for 20 instances. The average, minimum, and maximum gaps are $10.5 \%, 2.31 \%$, and $29.2 \%$, respectively.

Another formulation is given in Salhi et al [28] and Salhi and Rand [29]. They use flow variables to model capacities and subtour elimination constraints. Salhi and Rand mention the large size of the formulation. In both papers, no computation is reported with the formulation.

Different heuristics (see [6], [10], [11], [27], [28], [29], [30], and [33]) have been developed for the $H V R P$. As no good lower bounds are available, the measure of quality has been the improvement with respect to the previous best upper bound. The instances introduced in [12] are commonly used.

In this paper, we derive formulations and linear programming (LP) lower bounds for the $H V R P$. We limit ourselves to formulations where the capacity and subtour elimination constraints are modeled with Miller-Tucker-Zemlin (MTZ) constraints [24] or using flow variables.

We have four formulations based on MTZ constraints. As we go from the first formulation to the fourth, the size (number of variables and constraints) increases, so does the strength. Hence it is hard to conclude which formulation is better. When we compare the LP relaxations of these formulations on a small set of instances (four 20-node problems from [12]), we observe that they all give the same lower bound and the percentage gap is very high (minimum gap is $76.77 \%$ and maximum gap is $96.6 \%$ ). Clearly, such lower bounds are useless to comment on the quality of heuristic solutions.

We investigate why the lower bounds are so poor and then we strengthen all formulations through valid inequalities and lifting. The results are promising. For the instances mentioned above, the minimum gap becomes $1.11 \%$ and the maximum gap becomes $11.1 \%$.

We also consider two formulations based on flow variables. These formulations give better bounds compared to the first four formulations. We investigate which valid inequalities are implied by these formulations. We add the remaining valid inequalities to improve the lower bounds. With the strongest flow formulation, the minimum gap is $0.76 \%$ and the maximum gap is $4.9 \%$.

We compare the LP bounds of flow formulations and MTZ formulations through projection. Similar results exist for the Traveling Salesman Problem (TSP) (see e.g. in Gouveia and Pires [14], Langevin et al. [17], and Padberg and Sung [25]) and for the $C V R P$ (see Gouveia [13] and Letchford and Salazar-Gonzalez [20]).

The contribution of this paper is threefold. Different formulations are given for the $H V R P$ and lower bounds are derived for the problem instances from the literature. The well known subtour elimination inequalities, generalized large multistar inequalities and the lifting results for the MTZ constraints are generalized. Finally, the projection results for the MTZ and flow formulations are generalized.

This paper is organized as follows. In Section 2, we introduce the notation and state the assumptions. In Section 3, we derive the first four formulations, compare their LP 
bounds, strengthen them with valid inequalities and lifting. In Section 4, we present the two flow formulations and compare them with MTZ formulations. Then, we point out which valid inequalities are implied by these formulations. In Section 5, we present computational results. We conclude in Section 6.

\section{Preliminaries}

Node 0 stands for the origin. The set $N=\{1, \ldots, n\}$ is the set of demand nodes. Let $N_{0}=N \cup\{0\}$. A trip is said to end at node $i \in N$ if node $i$ is the last demand node of this trip. The demand of node $i \in N$ is denoted by $q_{i}$ and is a positive integer. We take $q_{0}=0$. For $S \subseteq N, q(S)=\sum_{i \in S} q_{i}$.

Let $K$ denote the set of different types of vehicles. For $k \in K$, let $F C_{k}$ denote the fixed cost of making a trip using vehicle type $k$ and $Q_{k}$ denote the capacity of vehicle $k$. For $i \in N$, let $K_{i}=\left\{k \in K: q_{i} \leq Q_{k}\right\}$; set $K_{i}$ is the set of vehicles that can serve node $i$. If $\cup_{i \in N} K_{i} \subset K$, then we remove all vehicle types in $K \backslash \cup_{i \in N} K_{i}$ from the problem. If for $k \in K, Q_{k}>q(N)$, then we replace $Q_{k}$ with $q(N)$. Let $q c$ be the greatest common divisor of $q_{1}, q_{2}, \ldots, q_{n}$. The total demand of nodes on a trip is then a multiple of $q c$. So for $k \in K$, we update the capacity of vehicle type $k$ to be $\left\lfloor\frac{Q_{k}}{q c}\right\rfloor q c$. If there are two vehicle types $k_{1}$ and $k_{2}$ with $Q_{k_{1}} \geq Q_{k_{2}}$ and $F C_{k_{1}} \leq F C_{k_{2}}$, then we remove vehicle type $k_{2}$. Without loss of generality, we assume that $Q_{1}>Q_{2}>\cdots>$ $Q_{|K|}$.

Let $A=\left\{(i, j): i \in N_{0}, j \in N \backslash\{i\}\right.$ such that $\left.q_{i}+q_{j} \leq Q_{1}\right\}$. The set $A$ does not include arcs from nodes in $N$ to the origin node. The reason is that once we know the last demand node on a trip, we know that the last arc of the trip is the arc from that node to the origin. We use this information in our formulations and do not need to consider the arcs from nodes of $N$ to the origin.

For two nodes $i \in N_{0}$ and $j \in N \backslash\{i\}$ that can be served by the same vehicle, $d_{i j}$ denotes the shortest distance from $i$ to $j$ and is the length of $\operatorname{arc}(i, j) \in A$. The distance matrix may be asymmetric. Note that the definition of the arc set $A$ implies knapsack cover inequalities (see e.g. Balas [3], Hammer et al. [15], and Wolsey [34]) for subsets of size 2 for vehicle type 1 .

For $(i, j) \in A$, let $K_{i j}=\left\{k \in K: q_{i}+q_{j} \leq Q_{k}\right\}$. Define also $A K=\{(i, j, k)$ : $\left.(i, j) \in A, k \in K_{i j}\right\}$.

For $i \in N$, if $q_{i}>Q_{1}$ then the problem is infeasible. If $q_{i} \leq Q_{1}$ and $q_{i}+q_{j}>Q_{1}$ for all $j \in N \backslash\{i\}$, then node $i$ is alone on a trip. As there is no availability constraint, we can find easily the cheapest way of making this trip and remove node $i$ from the problem. So in the sequel, we assume for all $i \in N$ that there exists a node $j \in N \backslash\{i\}$ such that $q_{i}+q_{j} \leq Q_{1}$, i.e., no node in the subgraph of $G=\left(N_{0}, A\right)$ induced by the node set $N$ is isolated.

Let $w_{F C}$ and $w_{T}$ be the weights of the fixed cost of trips and the total distance in the total cost respectively. For $i \in N$ and $k \in K_{i}$, let $C_{i k}=w_{F C} F C_{k}+w_{T} d_{i 0}$ where $d_{i 0}$ is the shortest path distance from node $i$ to the origin. 


\section{Formulations based on Miller-Tucker-Zemlin constraints}

In this section, we derive four formulations for the $H V R P$ using MTZ constraints. As we proceed, we either define new variables or disaggregate existing variables and obtain stronger formulations. Then, we derive valid inequalities and do lifting.

For the first formulation, we define $a_{i k}$ to be 1 if there is a trip that uses vehicle type $k \in K_{i}$ and that ends at node $i \in N$ and to be 0 otherwise, $x_{i j}$ to be 1 if $\operatorname{arc}(i, j) \in A$ is used and to be 0 otherwise, and $u_{i}$ to be the total demand of nodes on the trip till node $i$ (including node $i$ ).

Note that the variable $a_{i k}$ can be seen as the binary variable related to the arc from node $i$ to the origin and disaggregated by the vehicle type.

The formulation, called $H V R P_{1}$, is as follows:

$$
\begin{array}{ll}
\min & \sum_{i \in N} \sum_{k \in K_{i}} C_{i k} a_{i k}+w_{T} \sum_{(i, j) \in A} d_{i j} x_{i j} \\
\text { s.t. } & \sum_{j \in N} x_{0 j}=\sum_{i \in N} \sum_{k \in K_{i}} a_{i k} \\
& \sum_{j:(j, i) \in A} x_{j i}=1 \quad \forall i \in N \\
& \sum_{j:(i, j) \in A} x_{i j}=1-\sum_{k \in K_{i}} a_{i k} \quad \forall i \in N \\
& u_{j} \geq u_{i}+q_{j}-Q_{1}\left(1-x_{i j}\right) \quad \forall(i, j) \in A: i \neq 0 \\
& u_{i} \geq q_{i}+\sum_{j:(j, i) \in A} q_{j} x_{j i} \quad \forall i \in N \\
& u_{i} \leq \sum_{k \in K_{i}} Q_{k} a_{i k}+Q_{1}\left(1-\sum_{k \in K_{i}} a_{i k}\right) \\
& a_{i k} \in\{0,1\} \quad \forall i \in N \in K_{i} \\
& x_{i j} \in\{0,1\} \quad \forall(i, j) \in A .
\end{array}
$$

Because of constraints (4) and nonnegativity of $x_{i j}$ variables, for $i \in N, \sum_{k \in K_{i}} a_{i k} \leq$ 1, i.e., if node $i$ is the last demand node of a trip, this trip can be served by a single type of vehicle. Due to constraint (2), the number of arcs that go out of the origin node is equal to the number of trips. Constraints (3) ensure that for each demand node $i \in N$ there is an incoming arc and constraints (4) ensure that if no trip ends at node $i \in N$, then there is an arc going out of node $i$. If node $i$ is the last demand node of a trip, then no $\operatorname{arc}$ of $A$ goes out of node $i$.

Constraints (5) and (6) compute $u_{i}$ for each node. Because of constraints (5), subtours are eliminated. These constraints linearize requirements $u_{j} \geq\left(u_{i}+q_{j}\right) x_{i j}$ for all $(i, j) \in A$ such that $i \neq 0$. In (5), if $x_{i j}=1$, then $u_{j} \geq u_{i}+q_{j}$ as required. If $x_{i j}=0$, then $u_{j} \geq u_{i}+q_{j}-Q_{1}$. This is satisfied since $u_{j} \geq q_{j}$ and $u_{i} \leq Q_{1}$. Constraints (6) are due to Desrochers and Laporte [7] and replace weaker requirements $u_{i} \geq q_{i}$ for all $i \in N$.

Because of constraints (7), the sum of demands of nodes on the trip that ends at node $i$ cannot exceed the capacity of the vehicle which makes this trip. These are linearizations 
of requirements $u_{i} \sum_{k \in K_{i}} a_{i k} \leq \sum_{k \in K_{i}} Q_{k} a_{i k}$ for all $i \in N$. In (7), if $a_{i k}=1$ for some $k \in K_{i}$, then $u_{i} \leq Q_{k}$ as required. If $\sum_{k \in K_{i}} a_{i k}=0$, then $u_{i} \leq Q_{1}$ and this is valid.

The objective function (1) is the weighted sum of fixed cost of trips and the total distance.

Formulation $H V R P_{1}$ has $|A|+\sum_{i \in N}\left|K_{i}\right| 0-1$ and $n$ continuous variables and $|A|+3 n+1$ linear constraints.

Variables used in formulation $H V R P_{1}$ tell us the type of vehicle that serves a given node only if this node is the last node of a trip. Next, we derive another formulation, called $\mathrm{HVRP} \mathrm{P}_{2}$, which uses additional variables to carry similar information for intermediate nodes. We define $b_{i k}$ to be 1 if there is a trip that uses vehicle type $k \in K_{i}$ and that goes through node $i \in N$ but does not end at node $i$ and to be 0 otherwise.

Formulation $\mathrm{HVRP}_{2}$ is as follows: min (1) subject to (2)-(6), (8), (9), and

$$
\begin{aligned}
& \sum_{k \in K_{i}}\left(a_{i k}+b_{i k}\right)=1 \quad \forall i \in N \\
& u_{i} \leq \sum_{k \in K_{i}} Q_{k}\left(a_{i k}+b_{i k}\right) \quad \forall i \in N \\
& b_{i k} \in\{0,1\} \quad \forall i \in N, k \in K_{i} .
\end{aligned}
$$

Constraints (10) ensure that each node $i$ is served by one type of vehicle and constraints (11) state that the sum of demands of nodes on the trip that goes through node $i$ cannot exceed the capacity of the vehicle that serves this trip.

Formulation $H V R P_{2}$ has $|A|+2 \sum_{i \in N}\left|K_{i}\right| 0-1$ and $n$ continuous variables and $|A|+4 n+1$ linear constraints. It has the advantage that capacity constraints are initially linear. Indeed, these are stronger constraints.

Next, we disaggregate the flow variables by vehicle types. We say arc $(i, j, k) \in A K$ is used if there is a vehicle of type $k$ that goes directly from node $i$ to node $j$. We define $y_{i j k}$ to be 1 if arc $(i, j, k) \in A K$ is used and to be 0 otherwise.

The formulation below is called $\mathrm{HVRP}_{3}$ and is similar to the formulation given in Golden et al. [12].

$$
\begin{array}{ll}
\min & \sum_{i \in N} \sum_{k \in K_{i}} C_{i k} a_{i k}+w_{T} \sum_{(i, j, k) \in A K} d_{i j} y_{i j k} \\
& \quad \sum_{j:(0, j, k) \in A K} y_{0 j k}=\sum_{i \in N: k \in K_{i}} a_{i k} \quad \forall k \in K \\
& \sum_{j:(j, i, k) \in A K} y_{j i k}=a_{i k}+b_{i k} \quad \forall i \in N, k \in K_{i} \\
& \sum_{j:(i, j, k) \in A K} y_{i j k}=b_{i k} \quad \forall i \in N, k \in K_{i} \\
& u_{j} \geq u_{i}+q_{j}-\sum_{k \in K_{i}} Q_{k}\left(a_{i k}+b_{i k}\right)+\sum_{k \in K_{i j}} Q_{k} y_{i j k}
\end{array}
$$




$$
\begin{array}{ll}
u_{i} \geq q_{i}+\sum_{k \in K} \sum_{j:(j, i, k) \in A K} q_{j} y_{j i k} \quad \forall i \in N \\
y_{i j k} \in\{0,1\} \quad \forall(i, j, k) \in A K .
\end{array}
$$

Constraints (14) state that the number of arcs of type $k \in K$ that go out of the origin node is equal to the number of trips that use vehicle type $k$. Constraints (15) impose that there is an incoming arc of type $k$ to each node $i \in N$ which is visited by a vehicle of type $k \in K_{i}$. Constraints (16) ensure that if there is a trip that uses vehicle type $k \in K_{i}$ and that goes through node $i \in N$ but does not end at $i$, then there is an arc of type $k$ going out of node $i$. Otherwise, no arc of type $k$ goes out of node $i$. Constraints (17) linearize $u_{j} \geq\left(u_{i}+q_{j}\right) \sum_{k \in K_{i j}} y_{i j k}$. If $y_{i j k}=1$ for some $k$, then as $a_{i k}+b_{i k}=1,(17)$ simplifies to $u_{j} \geq u_{i}+q_{j}$ as required. If $\sum_{k \in K_{i j}} y_{i j k}=0$, then (17) is satisfied since $u_{j} \geq q_{j}$ and $u_{i} \leq \sum_{k \in K_{i}} Q_{k}\left(a_{i k}+b_{i k}\right)$. Constraints (18) are similar to (6).

Formulation $\mathrm{HVRP}_{3}$ has $|A K|+2 \sum_{i \in N}\left|K_{i}\right| 0-1$ and $n$ continuous variables and $2 n+|K|+|A|+2 \sum_{i \in N}\left|K_{i}\right|$ linear constraints. Constraints (17) are stronger than the corresponding constraints in [12].

Note that variables $a_{i k}$ and $b_{i k}$ can be eliminated using (16) first and then (15). We keep them for ease of presentation.

We can also disaggregate variables $u_{i}$. Define $v_{i k}$ to be the total demand of nodes on the trip that uses vehicle type $k \in K_{i}$ till node $i \in N$ (including node $i$ ).

Then the problem can be formulated as min (13) subject to (8), (10), (12), (14)-(16), (19), and

$$
\begin{aligned}
& v_{j k} \geq v_{i k}+q_{j}\left(a_{j k}+b_{j k}\right)-Q_{k}\left(a_{i k}+b_{i k}-y_{i j k}\right) \\
& v_{i k} \geq q_{i}\left(a_{i k}+b_{i k}\right)+\sum_{j:(j, i, k) \in A K} q_{j} y_{j i k} \quad \forall(i, j, k) \in A K: i \neq 0 \\
& v_{i k} \leq Q_{k}\left(a_{i k}+b_{i k}\right) \quad \forall i \in N, k \in K_{i}
\end{aligned}
$$

Constraints (20) and (21) compute $v_{i k}$ for each node $i \in N$ and vehicle type $k \in K_{i}$. Constraints (22) say that the sum of demands of nodes on the trip that uses vehicle type $k \in K_{i}$ and that goes through node $i$ cannot exceed the capacity of a vehicle of type $k$.

Constraints (20) eliminate subtours and are linearizations of nonlinear requirements $v_{j k} \geq\left(v_{i k}+q_{j}\right) y_{i j k}$ for all $(i, j, k) \in A K$ such that $i \neq 0$. If $y_{i j k}=1$, then $a_{i k}+b_{i k}=1$ and $a_{j k}+b_{j k}=1$. Then (20) is $v_{j k} \geq v_{i k}+q_{j}$. If $y_{i j k}=0$, then (20) is $v_{j k} \geq$ $v_{i k}+q_{j}\left(a_{j k}+b_{j k}\right)-Q_{k}\left(a_{i k}+b_{i k}\right)$ and is satisfied since $v_{j k} \geq q_{j}\left(a_{j k}+b_{j k}\right)$ and $v_{i k} \leq Q_{k}\left(a_{i k}+b_{i k}\right)$.

This formulation is called $H V R P_{4}$. It has $|A K|+2 \sum_{i \in N}\left|K_{i}\right| 0-1$ and $\sum_{i \in N}\left|K_{i}\right|$ continuous variables and $|A K|+3 \sum_{i \in N}\left|K_{i}\right|+|K|+n$ constraints.

As formulations $\mathrm{HVRP}_{3}$ and $\mathrm{HVRP}_{4}$ have disaggregated flow variables, they can be modified easily to handle different variable costs. This is not possible with formulations $H V R P_{1}$ and $H V R P_{2}$.

If $|K| \leq n$, then $H V R P_{1}$ and $H V R P_{2}$ have $O\left(n^{2}\right)$ variables and constraints. Formulation $H V R P_{3}$ has $O\left(n^{2}|K|\right)$ variables and $O\left(n^{2}\right)$ constraints. Finally $H V R P_{4}$ has $O\left(n^{2}|K|\right)$ variables and constraints. $H V R P_{1}$ and $H V R P_{2}$ have smaller sizes compared to the formulations given in [12], [28], and [29]. 


\subsection{Linear Programming Relaxations}

In this section, we compare the LP bounds of the four formulations. Let $L P_{i}$ denote the optimal value of the LP relaxation of formulation $H V R P_{i}$ for $i=1,2,3,4$.

Theorem 1. $L P_{1} \leq L P_{2} \leq L P_{3} \leq L P_{4}$.

Proof. To show that $L P_{2} \geq L P_{1}$, we need to show that constraints (11) imply constraints (7). The right hand side of constraint (11) is $\sum_{k \in K_{i}} Q_{k}\left(a_{i k}+b_{i k}\right)$ and is less than or equal to $\sum_{k \in K_{i}} Q_{k} a_{i k}+\sum_{k \in K_{i}} Q_{1} b_{i k}$. By constraints (10) this equals $\sum_{k \in K_{i}} Q_{k} a_{i k}+$ $Q_{1}\left(1-\sum_{k \in K_{i}} a_{i k}\right)$ which is the right hand side of constraint (7). So constraints (11) imply constraints (7).

Next we show that $L P_{3} \geq L P_{2}$. For $(a, b, y, u)$ feasible for the LP relaxation of $\mathrm{HVRP}_{3}$, consider $(a, b, x, u)$ where $x_{i j}=\sum_{k \in K_{i j}} y_{i j k}$ for all $(i, j) \in A$. We show that $(a, b, x, u)$ satisfies (5). The right hand side of constraint (17) is equal to $u_{i}+q_{j}-\sum_{k \in K_{i}} Q_{k}\left(a_{i k}+b_{i k}\right)+\sum_{k \in K_{i j}} Q_{k} y_{i j k}$. We can rewrite this as $u_{i}+q_{j}-$ $\sum_{k \in K_{i} \backslash K_{i j}} Q_{k}\left(a_{i k}+b_{i k}\right)-\sum_{k \in K_{i j}} Q_{k}\left(a_{i k}+b_{i k}-y_{i j k}\right)$. Now as $a_{i k}+b_{i k} \geq y_{i j k} \geq 0$, this is greater than or equal to $u_{i}+q_{j}-\sum_{k \in K_{i} \backslash K_{i j}} Q_{1}\left(a_{i k}+b_{i k}\right)-\sum_{k \in K_{i j}} Q_{1}\left(a_{i k}+b_{i k}-y_{i j k}\right)$ which simplifies to $u_{i}+q_{j}-Q_{1}+Q_{1} x_{i j}$. This last expression is the right hand side of constraint (5). It is easy to verify that $(a, b, x, u)$ satisfies the remaining constraints of the LP relaxation of $H V R P_{2}$. Also, the respective objective function values of these two solutions are the same.

Finally, for $(a, b, y, v)$ feasible for the LP relaxation of $H V R P_{4}$, we show that $(a, b, y, u)$ where $u_{i}=\sum_{k \in K_{i}} v_{i k}$ for all $i \in N$ is feasible for the LP relaxation of $H V R P_{3}$. We only prove that $(a, b, y, u)$ satisfies constraints (17); it is easy to prove that it satisfies the remaining constraints. We sum constraints (20) for a given $(i, j) \in A$ over $k \in K_{i j}$ to obtain $\sum_{k \in K_{i j}}\left(v_{j k}-q_{j}\left(a_{j k}+b_{j k}\right)\right) \geq \sum_{k \in K_{i j}}\left(v_{i k}-Q_{k}\left(a_{i k}+b_{i k}-y_{i j k}\right)\right)$. As $v_{j k}-q_{j}\left(a_{j k}+b_{j k}\right) \geq 0$ for all $k \in K_{j}$, the left hand side is less than or equal to $u_{j}-q_{j}=\sum_{k \in K_{j}}\left(v_{j k}-q_{j}\left(a_{j k}+b_{j k}\right)\right)$. The right hand side is greater than or equal to $u_{i}-\sum_{k \in K_{i}} Q_{k}\left(a_{i k}+b_{i k}\right)+\sum_{k \in K_{i j}} Q_{k} y_{i j k}$ since for $k \in K_{i}, v_{i k}-Q_{k}\left(a_{i k}+b_{i k}\right) \leq 0$. This proves that $L P_{4} \geq L P_{3}$.

Despite the result in Theorem 1, in the computational results (see Section 5), we observed in Table 1 that all four formulations give the same lower bound and that this bound is very poor. The reason is that as the fixed costs dominate the routing costs, in the optimal solution of the LP relaxations, $a_{i k}=0$ for all $i \in N$ and $k \in K_{i}$. In this case the origin node is not on any of the trips and the trips make fractional subtours.

The following proposition proves that in this case, all formulations have the same LP bound.

Proposition 1. If there exists an optimal solution to the $L P$ relaxation of $H V R P_{1}$ such that $a_{i k}=0$ for all $i \in N$ and $k \in K_{i}$, then $L P_{1}=L P_{2}=L P_{3}=L P_{4}$.

Proof. Let $(a, x, u)$ be an optimal solution to the LP relaxation of $H V R P_{1}$ such that $a_{i k}=0$ for all $i \in N$ and $k \in K_{i}$. Set $b_{i 1}=1$ and $b_{i k}=0$ for all $k \in K_{i} \backslash\{1\}$ and $i \in N$. Then $(a, b, x, u)$ is feasible for the LP relaxation of $H V R P_{2}$ and has the same objective function value as $(a, x, u)$ in $H V R P_{1}$. Set $y_{i j 1}=x_{i j}$ for all $(i, j) \in A$ and 
$y_{i j k}=0$ for all other $(i, j, k) \in A K$. Then $(a, b, y, u)$ is optimal for the LP relaxation of $H V R P_{3}$. Also $(a, b, y, v)$ where $v_{i 1}=u_{i}$ and $v_{i k}=0$ for all $k \in K_{i} \backslash\{1\}$ and $i \in N$ is optimal for the LP relaxation of $\mathrm{HVRP}_{4}$.

\subsection{Valid Inequalities}

3.2.1. Covering type inequalities In practice, we may encounter the case in Proposition 1 quite often. If it occurs, then clearly the lower bound of the LP relaxations is very poor. To improve this bound, we will add some valid inequalities of the form $\alpha_{a} a \geq \alpha_{0}+\alpha_{b} b$ where $\alpha_{a}, \alpha_{b}$ and $\alpha_{0}$ are all nonnegative.

Let $F_{i}$ be the feasible set of formulation $H V R P_{i}$ for $i=1, \ldots, 4$.

Consider the set $\Phi=\operatorname{conv}\left(\left\{z \in \mathbb{Z}_{+}^{|K|}: \sum_{k \in K} Q_{k} z_{k} \geq q(N)\right.\right.$ and $z_{k} \leq \mid\{i \in N$ : $\left.k \in K_{i}\right\} \mid$ for all $\left.k \in K\right\}$ ). Mazur [23] studies the polyhedral properties of the set without the upper bound constraints. Pochet and Wolsey [26] consider the same set as Mazur, but $Q_{k}$ 's are multiples of each other.

If $\sum_{k \in K} \alpha_{k} z_{k} \geq \alpha_{0}$ is a valid inequality for $\Phi$, then $\sum_{k \in K} \alpha_{k} \sum_{i \in N: k \in K_{i}} a_{i k} \geq \alpha_{0}$ is valid for $F_{i}$ for $i=1, \ldots, 4$.

Here we use the inequality

$$
\sum_{i \in N} \sum_{k \in K_{i}} Q_{k} a_{i k} \geq q(N)
$$

and apply Chvatal-Gomory procedure to obtain valid inequalities.

Proposition 2. For $Q>0$, the inequality

$$
\sum_{i \in N} \sum_{k \in K_{i}}\left\lceil\frac{Q_{k}}{Q}\right\rceil a_{i k} \geq\left\lceil\frac{q(N)}{Q}\right\rceil
$$

is valid for $F_{i}$ for $i=1, \ldots, 4$.

These inequalities define facets of $\operatorname{conv}\left(\left\{z \in \mathbb{Z}_{+}^{|K|}: \sum_{k \in K} Q_{k} z_{k} \geq q(N)\right\}\right)$ under some conditions, but they do not give the description of this polyhedron in general (Yaman [35]). For small sets $K$, one can generate all facet defining inequalities of $\Phi$ using PORTA [5] and use them to strengthen formulations of the HVRP.

It is possible to disaggregate inequality (23) using variables $b_{i k}$ 's. The following proposition is easy to prove.

Proposition 3. For $k \in K$, the inequality

$$
Q_{k} \sum_{i \in N: k \in K_{i}} a_{i k} \geq \sum_{i \in N: k \in K_{i}} q_{i}\left(a_{i k}+b_{i k}\right)
$$

is valid for $F_{2}, F_{3}$, and $F_{4}$. 
3.2.2. Subtour elimination inequalities If inequality $\sum_{(i, j) \in A} \alpha_{i j} x_{i j} \leq \alpha_{0}$ is a valid inequality for the $C V R P$ where each vehicle has capacity $Q_{1}$, then it is also valid for $F_{1}$ and $F_{2}$ and $\sum_{(i, j) \in A} \alpha_{i j} \sum_{k \in K_{i j}} y_{i j k} \leq \alpha_{0}$ is valid for $F_{3}$ and $F_{4}$. So we can use known valid inequalities of the $C V R P$ to strengthen our formulations.

One famous family is the family of subtour elimination inequalities. Let $(S: T)=$ $\{(i, j) \in A: i \in S, j \in T\}$,

$$
X(S: T)=\sum_{(i, j) \in(S: T)} x_{i j},
$$

and

$$
Y(S: T)=\sum_{(i, j) \in(S: T)} \sum_{k \in K_{i j}} y_{i j k} .
$$

For $S \subseteq N$, inequality

$$
X(S: S) \leq|S|-\left\lceil\frac{q(S)}{Q_{1}}\right\rceil
$$

is valid for $F_{1}$ and $F_{2}$, and inequality

$$
Y(S: S) \leq|S|-\left\lceil\frac{q(S)}{Q_{1}}\right\rceil
$$

is valid for $F_{3}$ and $F_{4}$.

Inequalities (26) and (27) for $S=N$ are the same as inequality (23) for $Q=Q_{1}$.

For the $C V R P$, replacing constraints (5)-(7) with (26), we obtain a valid formulation. These constraints eliminate subtours and impose capacity restrictions. To obtain such a formulation for the $H V R P$, we need to disaggregate inequalities (27). For $k \in K$, let $Y^{k}(S: T)=\sum_{(i, j) \in(S: T): k \in K_{i j}} y_{i j k}$.

Proposition 4. For $k \in K$ and $S \subseteq\left\{i \in N: k \in K_{i}\right\}$, the inequality

$$
Y^{k}(S: S) \leq|S|-\left\lceil\frac{q(S)}{Q_{k}}\right\rceil
$$

is valid for $F_{3}$ and $F_{4}$.

Proof. Let $S^{\prime}=\left\{i \in S: a_{i k}+b_{i k}=1\right\}$ and let $\bar{S}=S \backslash S^{\prime}$. Then $Y^{k}\left(S^{\prime}: S^{\prime}\right) \leq$ $\left|S^{\prime}\right|-\left\lceil\frac{q\left(S^{\prime}\right)}{Q_{k}}\right\rceil$ and the left hand side of inequality (28) is equal to $Y^{k}\left(S^{\prime}: S^{\prime}\right)$. We should show that $\left|S^{\prime}\right|-\left\lceil\frac{q\left(S^{\prime}\right)}{Q_{k}}\right\rceil \leq|S|-\left\lceil\frac{q(S)}{Q_{k}}\right\rceil$. Since $|\bar{S}|-\left\lceil\frac{q(S)}{Q_{k}}\right\rceil+\left\lceil\frac{q\left(S^{\prime}\right)}{Q_{k}}\right\rceil$ is greater than or equal to $|\bar{S}|-\left\lceil\frac{q(\bar{S})}{Q_{k}}\right\rceil$, and as $q_{i} \leq Q_{k}$ for all $i \in \bar{S}$, we have $|\bar{S}|-\left\lceil\frac{q(\bar{S})}{Q_{k}}\right\rceil \geq 0$ and so $|\bar{S}|-\left\lceil\frac{q(S)}{Q_{k}}\right\rceil+\left\lceil\frac{q\left(S^{\prime}\right)}{Q_{k}}\right\rceil \geq 0$. 
Replacing (11), (17), and (18) in $H V R P_{3}$ and (20)-(22) in $H V R P_{4}$ with inequalities (28) for all $k \in K$ and $S \subseteq\left\{i \in N: k \in K_{i}\right\}$, we obtain a valid formulation for the $H V R P$.

Replacing constraints (5)-(7) in formulation $H V R P_{1}$ and (5), (6), and (11) in $H V R P_{2}$ with inequalities (26) for all $S \subseteq N$ does not give a formulation. But one can obtain a valid formulation using inequalities $X\left(N_{0} \backslash S: S\right) \geq\left\lceil\frac{q(S)}{Q_{k}}\right\rceil\left(\sum_{i \in S}\left(a_{i k}+\right.\right.$ $\left.\left.b_{i k}\right)-|S|+1\right)$ for all $k \in K$ and $S \subseteq\left\{i \in N: k \in K_{i}\right\}$.

Note also that while inequalities (26) and (27) are equivalent to $X\left(N_{0} \backslash S: S\right) \geq$ $\left\lceil\frac{q(S)}{Q_{1}}\right\rceil$ and $Y\left(N_{0} \backslash S: S\right) \geq\left\lceil\frac{q(S)}{Q_{1}}\right\rceil$, the disaggregated inequality $Y^{k}\left(N_{0} \backslash S: S\right) \geq$ $\left\lceil\frac{q(S)}{Q_{k}}\right\rceil$ is not valid. It can be modified as $Y^{k}\left(N_{0} \backslash S: S\right) \geq\left\lceil\frac{q(S)}{Q_{k}}\right\rceil\left(\sum_{i \in S}\left(a_{i k}+\right.\right.$ $\left.\left.b_{i k}\right)-|S|+1\right)$ to be valid. Indeed, for $K^{\prime} \subseteq K$, inequality $\sum_{k \in K^{\prime}} Y^{k}\left(N_{0} \backslash S: S\right) \geq$ $\left\lceil\frac{q(S)}{Q^{\prime}}\right\rceil\left(\sum_{i \in S} \sum_{k \in K^{\prime} \cap K_{i}}\left(a_{i k}+b_{i k}\right)-|S|+1\right)$ where $Q^{\prime}=\max _{k \in K^{\prime}} Q_{k}$ is a valid inequality. For $K^{\prime}=K$, we obtain inequality $Y\left(N_{0} \backslash S: S\right) \geq\left\lceil\frac{q(S)}{Q_{1}}\right\rceil$.

For $K^{\prime} \subseteq K$ and $Q^{\prime}=\max _{k \in K^{\prime}} Q_{k}$, inequality $\sum_{k \in K^{\prime}} Y^{k}(S: S) \leq|S|-\left\lceil\frac{q(S)}{Q^{\prime}}\right\rceil$ is also a valid inequality. If $\left\lceil\frac{q(S)}{Q^{\prime}}\right\rceil=\left\lceil\frac{q(S)}{Q_{k}}\right\rceil$ for all $k \in K^{\prime}$, then this inequality dominates inequalities (28) for $k \in K^{\prime}$.

Next, we improve inequalities (27) for subsets $S$ such that $q(S) \leq Q_{1}$.

Theorem 2. For $S \subseteq N$ such that $|S| \geq 3$ and $q(S) \leq Q_{1}$, the inequality

$$
\sum_{k \in K: q(S) \leq Q_{k}} Y^{k}(S: S)+\frac{|S|-1}{|S|-2} \sum_{k \in K: q(S)>Q_{k}} Y^{k}(S: S) \leq|S|-1
$$

is valid for $F_{3}$ and $F_{4}$.

Proof. If $\sum_{k \in K: q(S)>Q_{k}} Y^{k}(S: S)=0$, then inequality (29) reduces to the valid inequality $\sum_{k \in K: q(S) \leq Q_{k}} Y^{k}(S: S) \leq|S|-1$. If $\sum_{k \in K: q(S) \leq Q_{k}} Y^{k}(S: S)=0$, then inequality $\sum_{k \in K: q(S)>Q_{k}} Y^{k}(S: S) \leq|S|-2$ is valid and is the same as (29). Finally, if $\sum_{k \in K: q(S)>Q_{k}} Y^{k}(S: S)>0$ and $\sum_{k \in K: q(S) \leq Q_{k}} Y^{k}(S: S)>0$, then $\sum_{k \in K: q(S)>Q_{k}} Y^{k}(S: S)+\sum_{k \in K: q(S) \leq Q_{k}} Y^{k}(S: S) \leq|S|-2$ is valid and dominates (29). So inequality (29) is valid.

If $S$ is such that $q(S) \leq Q_{1}$, inequality (29) dominates inequalities (27). If, in addition $\left\lceil\frac{q(S)}{Q_{k}}\right\rceil \leq 2$ for all $k \in K$, inequality (29) dominates inequalities (28). In this case, it is better to use inequality (29) rather than inequalities (28) not only because it dominates but also because we use one inequality instead of $|K|$ inequalities.

3.2.3. Generalized large multistar inequalities Generalized large multistar inequalities are valid for the $C V R P$ (see Araque et al. [2], Gouveia [13], Letchford and Salazar-Gonzalez [20], and Letchford et al. [21]). For $S \subseteq N$, inequality

$$
\sum_{(i, j) \in\left(N_{0} \backslash S: S\right)}\left(Q_{1}-q_{i}\right) x_{i j} \geq q(S)+\sum_{(i, j) \in(S: N \backslash S)} q_{j} x_{i j}
$$


is valid for $F_{1}$ and $F_{2}$, and inequality

$$
\sum_{(i, j) \in\left(N_{0} \backslash S: S\right)}\left(Q_{1}-q_{i}\right) \sum_{k \in K_{i j}} y_{i j k} \geq q(S)+\sum_{(i, j) \in(S: N \backslash S)} \sum_{k \in K_{i j}} q_{j} y_{i j k}
$$

is valid for $F_{3}$ and $F_{4}$.

These inequalities imply that the remaining capacity on the vehicles entering nodes of $S$ should be at least the demand of $S$ and the nodes that come immediately after $S$. Inequalities (31) can be improved and disaggregated for the $H V R P$ as follows:

Proposition 5. For $S \subseteq N$, the inequality

$$
\sum_{(i, j) \in\left(N_{0} \backslash S: S\right)} \sum_{k \in K_{i j}}\left(Q_{k}-q_{i}\right) y_{i j k} \geq q(S)+\sum_{(i, j) \in(S: N \backslash S)} \sum_{k \in K_{i j}} q_{j} y_{i j k}
$$

and for $k \in K$,

$$
\begin{aligned}
\sum_{(i, j) \in\left(N_{0} \backslash S: S\right): k \in K_{i j}}\left(Q_{k}-q_{i}\right) y_{i j k} \geq & \sum_{j \in S: k \in K_{j}} q_{j}\left(a_{j k}+b_{j k}\right) \\
& +\sum_{(i, j) \in(S: N \backslash S): k \in K_{i j}} q_{j} y_{i j k}
\end{aligned}
$$

are valid for $F_{3}$ and $F_{4}$.

For $S=N$, inequality (32) is the same as inequality (23) and for $k \in K$, inequality (33) is the same as inequality (25).

Notice that for small subsets $S$, if $q(S)$ is much smaller than $Q_{1}$, inequality (30) may not be strong. The same is true for inequalities (32) and (33). Indeed, coefficients of the variables can be improved as follows:

Proposition 6. For $S \subseteq N$, let $\delta(S)=\{j \in N \backslash S: \exists(i, j) \in A$ with $i \in S\}$. The inequality

$$
\sum_{(i, j) \in\left(N_{0} \backslash S: S\right)} \min \left\{Q_{1}-q_{i}, q(S)+\max _{m \in \delta(S)} q_{m}\right\} x_{i j} \geq q(S)+\sum_{(i, j) \in(S: N \backslash S)} q_{j} x_{i j}
$$

is valid for $F_{1}$ and $F_{2}$. For $k \in K$, let $\delta^{k}(S)=\{j \in N \backslash S: \exists(i, j, k) \in A K$ with $i \in S\}$. Also let $\sigma_{i k}=\min \left\{Q_{k}-q_{i}, q(S)+\max _{m \in \delta^{k}(S)} q_{m}\right\}$ for $i \in N_{0} \backslash S$ and $k \in K_{i}$. Then the inequalities

$$
\sum_{(i, j) \in\left(N_{0} \backslash S: S\right)} \sum_{k \in K_{i j}} \sigma_{i k} y_{i j k} \geq q(S)+\sum_{(i, j) \in(S: N \backslash S)} \sum_{k \in K_{i j}} q_{j} y_{i j k}
$$

and for $k \in K$,

$$
\sum_{(i, j) \in\left(N_{0} \backslash S: S\right): k \in K_{i j}} \sigma_{i k} y_{i j k} \geq \sum_{j \in S: k \in K_{j}} q_{j}\left(a_{j k}+b_{j k}\right)+\sum_{(i, j) \in(S: N \backslash S): k \in K_{i j}} q_{j} y_{i j k}
$$

are valid for $F_{3}$ and $F_{4}$. 
Proof. We prove the validity of inequality (34). The proof is similar for the other inequalities. For $S \subseteq N$, let $A_{1}=\left\{(i, j) \in\left(N_{0} \backslash S: S\right): Q_{1}-q_{i}<q(S)+\max _{m \in \delta(S)} q_{m}\right.$ and $\left.x_{i j}=1\right\}$ and $A_{2}=\left\{(i, j) \in\left(N_{0} \backslash S: S\right): Q_{1}-q_{i} \geq q(S)+\max _{m \in \delta(S)} q_{m}\right.$ and $\left.x_{i j}=1\right\}$. If $A_{2}=\emptyset$ then inequality (34) is the same as inequality (30) and so is satisfied. Now suppose that $A_{2} \neq \emptyset$. Let $S_{1}$ be the set of nodes in $S$ served by trips entering $S$ using arcs in $A_{1}$. Then $\sum_{(i, j) \in A_{1}}\left(Q_{1}-q_{i}\right) \geq q\left(S_{1}\right)+\sum_{(i, j) \in\left(S_{1}: N \backslash S\right)} q_{j} x_{i j}$ should be satisfied. Similarly, let $S_{2}$ be the set of nodes in $S$ served by trips entering $S$ using arcs in $A_{2}$. It remains to show that $\left|A_{2}\right|\left(q(S)+\max _{m \in \delta(S)} q_{m}\right) \geq q\left(S_{2}\right)+\sum_{(i, j) \in\left(S_{2}: N \backslash S\right)} q_{j} x_{i j}$. Since $X\left(S_{2}: N \backslash S\right) \leq\left|A_{2}\right|$, and as $\left|A_{2}\right| \geq 1$, this is satisfied.

Observe that inequalities (34), (35), and (36) dominate inequalities (30), (32), and (33) respectively.

\subsection{Lifting of the MTZ constraints}

Next, we strengthen some of the constraints in the formulations. Our results are adaptations of the lifting results in Desrochers and Laporte [7] and Kara et al. [16].

3.3.1. Formulation $H V R P_{1}$ In $H V R P_{1}$, by definition, we want $u_{j}$ to be $u_{i}+q_{j}$ if $x_{i j}=1$. In other words, we want

$$
u_{j}=\sum_{i:(i, j) \in A}\left(u_{i}+q_{j}\right) x_{i j} \text { for all } j \in N .
$$

But our formulation may have solutions which do not satisfy this requirement. Let $0 \rightarrow i_{1} \rightarrow \ldots \rightarrow i_{m}$ be a trip done by vehicle type $k$. Let $u$ be a vector which satisfies (37). If $u_{i_{m}}<Q_{k}$, then a vector $u^{\prime}$ which is the same as $u$ except that $u_{i_{m}}^{\prime}=Q_{k}$ is also feasible.

Still there exists always an optimal solution which satisfies the requirement of the definition. We call such an optimal solution a tight optimal solution and present inequalities that it satisfies.

Proposition 7. A tight optimal solution satisfies the inequality

$$
u_{j} \geq u_{i}+q_{j}-Q_{1}\left(1-x_{i j}\right)+\sum_{k \in K^{\prime}}\left(Q_{1}-Q_{k}\right) a_{i k}+\left(\min _{k \in K^{\prime} \cap K_{i j}} Q_{k}-q_{i}-q_{j}\right) x_{j i}
$$

for $(i, j) \in A$ such that $i \neq 0$ and $K^{\prime} \subseteq K_{i}$.

Proof. Suppose that $\sum_{k \in K^{\prime}} a_{i k}=0$. If $x_{j i}=0$ then inequality (38) is the same as inequality (5). If $x_{j i}=1$, then $x_{i j}=0$ and a tight optimal solution satisfies $u_{i}=u_{j}+q_{i}$. This solution also satisfies inequality (38).

Now suppose that $a_{i k^{\prime}}=1$ for some $k^{\prime} \in K^{\prime}$. Then $x_{i j}=0$. If $x_{j i}=0$, inequality (38) reduces to $u_{j} \geq u_{i}+q_{j}-Q_{k^{\prime}}$ which is satisfied since $u_{j} \geq q_{j}$ and $u_{i} \leq Q_{k^{\prime}}$. If $x_{j i}=1$, inequality (38) reduces to $u_{j} \geq u_{i}-Q_{k^{\prime}}+\min _{k \in K^{\prime} \cap K_{i j}} Q_{k}-q_{i}$. A tight optimal solution satisfies $u_{i}=u_{j}+q_{i}$. Moreover, as $x_{j i}=1, k^{\prime} \in K_{i j}$. So, this optimal solution satisfies the above inequality. 
For $(i, j) \in A$ such that $i \neq 0$ and $K^{\prime}=\{1\}$, we obtain inequality

$$
u_{j} \geq u_{i}+q_{j}-Q_{1}\left(1-x_{i j}\right)+\left(Q_{1}-q_{i}-q_{j}\right) x_{j i}
$$

which dominates constraint (5) since $Q_{1}-q_{i}-q_{j} \geq 0$.

Proposition 8. A tight optimal solution satisfies

$$
\begin{aligned}
u_{i} \leq & \sum_{k \in K_{i}} Q_{k} a_{i k}+Q_{1}\left(1-\sum_{k \in K_{i}} a_{i k}\right)-\sum_{j:(i, j) \in A} q_{j} x_{i j} \\
& -\min \left\{Q_{1}-q_{i}-\max _{j:(i, j) \in A} q_{j}, \min _{k \in K_{i}} Q_{k}-q_{i}\right\} x_{0 i}
\end{aligned}
$$

for $i \in N$.

Proof. Suppose that $x_{i j^{\prime}}=1$ for some $\left(i, j^{\prime}\right) \in A$. Then $\sum_{k \in K_{i}} a_{i k}=0$ and inequality (40) simplifies to $u_{i} \leq Q_{1}-q_{j^{\prime}}-\min \left\{Q_{1}-q_{i}-\max _{j:(i, j) \in A} q_{j}, \min _{k \in K_{i}} Q_{k}-q_{i}\right\} x_{0 i}$. If $x_{0 i}=0$, then $u_{i} \leq Q_{1}-q_{j^{\prime}}$ is valid since $u_{j^{\prime}} \geq u_{i}+q_{j^{\prime}}$ and $u_{j^{\prime}} \leq Q_{1}$. If $x_{0 i}=1$, then a tight optimal solution satisfies $u_{i}=q_{i}$ and so also $u_{i} \leq-q_{j^{\prime}}+q_{i}+$ $\max _{j:(i, j) \in A} q_{j}$. The right hand side is less than or equal to $Q_{1}-q_{j^{\prime}}-\min \left\{Q_{1}-q_{i}-\right.$ $\left.\max _{j:(i, j) \in A} q_{j}, \min _{k \in K_{i}} Q_{k}-q_{i}\right\}$.

Now suppose that $\sum_{j:(i, j) \in A} x_{i j}=0$. Then $a_{i k^{\prime}}=1$ for some $k^{\prime} \in K_{i}$. Inequality (40) simplifies to $u_{i} \leq Q_{k^{\prime}}-\min \left\{Q_{1}-q_{i}-\max _{j:(i, j) \in A} q_{j}, \min _{k \in K_{i}} Q_{k}-q_{i}\right\} x_{0 i}$. If $x_{0 i}=0$, then $u_{i} \leq Q_{k^{\prime}}$ is valid. If $x_{0 i}=1$, then a tight optimal solution satisfies $u_{i}=q_{i}$. So it also satisfies $u_{i} \leq Q_{k^{\prime}}-\min _{k \in K_{i}} Q_{k}+q_{i}$ which is less than or equal to $Q_{k^{\prime}}-\min \left\{Q_{1}-q_{i}-\max _{j:(i, j) \in A} q_{j}, \min _{k \in K_{i}} Q_{k}-q_{i}\right\}$.

Inequalities (40) dominate constraints (7) since $q_{j} \geq 0$ for all $j$ such that $(i, j) \in A$, $Q_{1}-q_{i}-\max _{j:(i, j) \in A} q_{j} \geq 0$ and $\min _{k \in K_{i}} Q_{k}-q_{i} \geq 0$.

If $|K|=1$, then inequalities (38) and (39) simplify to

$$
u_{j} \geq u_{i}+q_{j}-Q_{1}\left(1-x_{i j}\right)+\left(Q_{1}-q_{i}-q_{j}\right) x_{j i}
$$

and inequality (40) simplifies to

$$
u_{i} \leq Q_{1}-\sum_{j:(i, j) \in A} q_{j} x_{i j}-\left(Q_{1}-q_{i}-\max _{j \in N \backslash\{i\}: q_{i}+q_{j} \leq Q_{1}} q_{j}\right) x_{0 i} .
$$

Both inequalities are given in Kara et al. [16].

We replace constraints (5) by (39) and constraints (7) by (40) in $H V R P_{1}$.

3.3.2. Formulation $H V R P_{2} \quad$ Constraints (10) in formulation $H V R P_{2}$ can be strengthened as follows:

Proposition 9. A tight optimal solution satisfies

$$
\begin{aligned}
u_{i} \leq & \sum_{k \in K_{i}} Q_{k}\left(a_{i k}+b_{i k}\right)-\sum_{j:(i, j) \in A} q_{j} x_{i j} \\
& -\min \left\{\min _{j:(i, j) \in A}\left\{\min _{k \in K_{i j}} Q_{k}-q_{i}-q_{j}\right\}, \min _{k \in K_{i}} Q_{k}-q_{i}\right\} x_{0 i}
\end{aligned}
$$

for $i \in N$. 
Proof. If $x_{0 i}=0$, then the resulting inequality is valid. Now suppose that $x_{0 i}=1$. So a tight optimal solution has $u_{i}=q_{i}$. Let $k^{\prime} \in K_{i}$ be such that $a_{i k^{\prime}}+b_{i k^{\prime}}=1$. We investigate two cases. If $\sum_{j:(i, j) \in A} x_{i j}=0$, then a tight optimal solution has $u_{i}=q_{i}$ and so satisfies $q_{i} \leq Q_{k^{\prime}}-\min _{k \in K_{i}} Q_{k}+q_{i}$ which is less than or equal to $Q_{k^{\prime}}-$ $\min \left\{\min _{j:(i, j) \in A}\left\{\min _{k \in K_{i j}} Q_{k}-q_{i}-q_{j}\right\}, \min _{k \in K_{i}} Q_{k}-q_{i}\right\}$.

If $x_{i j^{\prime}}=1$ for some $\left(i, j^{\prime}\right) \in A$, since $Q_{k^{\prime}}-q_{j^{\prime}} \geq \min _{k \in K_{i j}} Q_{k}-q_{j^{\prime}}$ and $\min _{k \in K_{i j}{ }^{\prime}} Q_{k}-q_{j^{\prime}} \geq \min _{j:(i, j) \in A}\left\{\min _{k \in K_{i j}} Q_{k}-q_{j}\right\}$, a tight optimal solution satisfies $u_{i}=q_{i}$. Then $q_{i} \leq Q_{k^{\prime}}-q_{j^{\prime}}-\min _{j:(i, j) \in A}\left\{\min _{k \in K_{i j}} Q_{k}-q_{i}-q_{j}\right\}$ which is less than or equal to $Q_{k^{\prime}}-\min \left\{\min _{j:(i, j) \in A}\left\{\min _{k \in K_{i j}} Q_{k}-q_{i}-q_{j}\right\}, \min _{k \in K_{i}} Q_{k}-q_{i}\right\}$.

3.3.3. Formulation $H V R P_{3}$ Next we present two families of inequalities that dominate (17) and (11) in $H V R P_{3}$.

Proposition 10. For $(i, j) \in A$ such that $i \neq 0$, a tight optimal solution satisfies

$$
\begin{gathered}
u_{j} \geq u_{i}+q_{j}-\sum_{k \in K_{i}} Q_{k}\left(a_{i k}+b_{i k}\right)+\sum_{k \in K_{i j}} Q_{k} y_{i j k} \\
+\sum_{k:(j, i, k) \in A K}\left(Q_{k}-q_{i}-q_{j}\right) y_{j i k} .
\end{gathered}
$$

Proof. If $y_{j i k}=1$ for some $j$ and $k$ such that $(j, i, k) \in A K$, then $\sum_{k \in K_{i j}} y_{i j k}=0$, and $a_{i k}+b_{i k}=1$. So inequality (42) simplifies to $u_{j} \geq u_{i}-q_{i}$. An optimal solution which satisfies $u_{i}=u_{j}+q_{i}$ satisfies the inequality.

Proposition 11. A tight optimal solution satisfies

$$
\begin{array}{r}
u_{i} \leq \sum_{k \in K_{i}} Q_{k}\left(a_{i k}+b_{i k}\right)-\sum_{k \in K} \sum_{j:(i, j, k) \in A K} q_{j} y_{i j k} \\
\sum_{k \in K}\left(Q_{k}-q_{i}-\max _{j:(i, j, k) \in A K} q_{j}\right) y_{0 i k}
\end{array}
$$

for $i \in N$.

Proof. If $y_{i j^{\prime} k^{\prime}}=1$ for some $\left(i, j^{\prime}, k^{\prime}\right) \in A K$, then $a_{i k^{\prime}}=0$ and $b_{i k^{\prime}}=1$. Inequality (43) simplifies to $u_{i} \leq Q_{k^{\prime}}-q_{j^{\prime}}-\left(Q_{k^{\prime}}-q_{i}-\max _{j:\left(i, j, k^{\prime}\right) \in A K} q_{j}\right) y_{0 i k^{\prime}}$. If $y_{0 i k^{\prime}}=0$, then $u_{i} \leq Q_{k^{\prime}}-q_{j^{\prime}}$ is satisfied since $u_{j^{\prime}} \geq u_{i}+q_{j^{\prime}}$ and $u_{j^{\prime}} \leq Q_{k^{\prime}}$. If $y_{0 i k^{\prime}}=1$, then a tight optimal solution satisfies $u_{i} \leq-q_{j^{\prime}}+q_{i}+\max _{j:\left(i, j, k^{\prime}\right) \in A K} q_{j}$.

If $\sum_{k \in K} \sum_{j:(i, j, k) \in A K} y_{i j k}=0$, then $a_{i k^{\prime}}=1$ for some $k^{\prime} \in K_{i}$. Inequality (43) simplifies to $u_{i} \leq Q_{k^{\prime}}-\left(Q_{k^{\prime}}-q_{i}-\max _{j:\left(i, j, k^{\prime}\right) \in A K} q_{j}\right) y_{0 i k^{\prime}}$. If $y_{0 i k^{\prime}}=0$, then $u_{i} \leq Q_{k^{\prime}}$ is satisfied. Otherwise, inequality (43) becomes $u_{i} \leq q_{i}+\max _{j:\left(i, j, k^{\prime}\right) \in A K} q_{j}$. A tight optimal solution satisfies this inequality.

3.3.4. Formulation $\mathrm{HVRP}_{4}$ Finally, we strengthen constraints (20) and (22) in formulation $\mathrm{HVRP}_{4}$.

Proposition 12. For $(i, j, k) \in A K$, a tight optimal solution satisfies

$$
v_{j k} \geq v_{i k}+q_{j}\left(a_{j k}+b_{j k}\right)-Q_{k}\left(a_{i k}+b_{i k}-y_{i j k}\right)+\left(Q_{k}-q_{i}-q_{j}\right) y_{j i k} \text {. }
$$


Proof. If $y_{j i k}=1$ then $y_{i j k}=0, a_{j k}+b_{j k}=1$, and $a_{i k}+b_{i k}=1$. So inequality (44) becomes $v_{j k} \geq v_{i k}-q_{i}$. As $y_{j i k}=1$, a tight optimal solution satisfies $v_{i k}=v_{j k}+q_{i}$.

\section{Proposition 13. A tight optimal solution satisfies}

$$
v_{i k} \leq Q_{k}\left(a_{i k}+b_{i k}\right)-\sum_{j:(i, j, k) \in A K} q_{j} y_{i j k}-\left(Q_{k}-q_{i}-\max _{j:(i, j, k) \in A K} q_{j}\right) y_{0 i k}
$$

for $i \in N$.

Proof. If $y_{i j^{\prime} k}=1$ for some $\left(i, j^{\prime}, k\right) \in A K$, then $a_{i k}=0$ and $b_{i k}=1$. Inequality (45) simplifies to $v_{i k} \leq Q_{k}-q_{j^{\prime}}-\left(Q_{k}-q_{i}-\max _{j:(i, j, k) \in A K} q_{j}\right) y_{0 i k}$. If $y_{0 i k}=0$, then $v_{i k} \leq Q_{k}-q_{j^{\prime}}$ is satisfied since $v_{j^{\prime} k} \geq v_{i k}+q_{j^{\prime}}$ and $v_{j^{\prime} k} \leq Q_{k}$. If $y_{0 i k}=1$, then $v_{i k} \leq-q_{j^{\prime}}+q_{i}+\max _{j:(i, j, k) \in A K} q_{j}$ is satisfied by a tight optimal solution.

If $\sum_{j:(i, j, k) \in A K} y_{i j k}=0$, then $b_{i k}=0$. If $a_{i k}=1, v_{i k} \leq Q_{k}-\left(Q_{k}-q_{i}-\right.$ $\left.\max _{j:(i, j, k) \in A K} q_{j}\right) y_{0 i k}$. If $y_{0 i k}=0$, then $v_{i k} \leq Q_{k}$ is satisfied. Otherwise, $v_{i k} \leq$ $q_{i}+\max _{j:(i, j, k) \in A K} q_{j}$. A tight optimal solution satisfies this inequality. If $a_{i k}=0$, then $y_{0 i k}=0$. Inequality (45) simplifies to $v_{i k} \leq 0$ which is already implied by (22).

\section{Flow Formulations}

In this section, we present two formulations where the capacity constraints and the subtour elimination constraints are expressed using flows (see e.g. Baldacci et al. [4], Gavish and Graves [9], Gouveia [13], and Letchford and Salazar-Gonzalez [20] for flow formulations for the $C V R P$ ). We compare the LP bounds with those of MTZ formulations and study some of the valid inequalities presented in the previous section.

We can consider the demand requirements in the following way. The origin node sends $q_{i}$ units of flow to each node $i \in N$. Let $f_{i j}$ be the flow on arc $(i, j) \in A$. Using these additional variables, we can formulate the $H V R P$ as follows: min (13) subject to (8), (10), (12), (14)-(16), (19), and

$$
\begin{aligned}
& \sum_{j:(j, i) \in A} f_{j i}-\sum_{j:(i, j) \in A} f_{i j}=q_{i} \quad \forall i \in N \\
& f_{i j} \leq \sum_{k \in K_{i j}}\left(Q_{k}-q_{i}\right) y_{i j k} \quad \forall(i, j) \in A \\
& f_{i j} \geq \sum_{k \in K_{i j}} q_{j} y_{i j k} \quad \forall(i, j) \in A .
\end{aligned}
$$

Let $H V R P_{5}$ be the above formulation. Constraints (46) imply that $q_{i}$ units of flow should be sent to node $i \in N$. Summing up these constraints, we obtain $\sum_{i \in N} f_{0 i}=$ $q(N)$; so the origin node is sending a total of $q(N)$ units. If arc $(i, j)$ is traversed by a vehicle of type $k$, then, due to constraints (47), flow from node $i$ to node $j$ plus the demand of node $i$ cannot exceed the capacity of the vehicle. If $\operatorname{arc}(i, j)$ is used then the flow on it should be at least the demand of node $j$ due to constraints (48). 
Formulation $H V R P_{5}$ has $|A K|+2 \sum_{i \in N}\left|K_{i}\right| 0-1$ and $|A|$ continuous variables and $2\left(|A|+n+\sum_{i \in N}\left|K_{i}\right|\right)+|K|$ linear constraints. This formulation is similar to the one given in Salhi et al [28] and Salhi and Rand [29]. But constraints (47) and (48) are stronger than their corresponding ones.

Next by disaggregating variables $f_{i j}$, we obtain another formulation. Formulation $H V R P_{6}$ is as follows: min (13) subject to (8), (10), (12), (14)-(16), (19), and

$$
\begin{aligned}
& \sum_{j:(j, i) \in A} g_{j i k}-\sum_{j:(i, j) \in A} g_{i j k}=q_{i}\left(a_{i k}+b_{i k}\right) \quad \forall i \in N, k \in K_{i} \\
& g_{i j k} \leq\left(Q_{k}-q_{i}\right) y_{i j k} \quad \forall(i, j, k) \in A K \\
& g_{i j k} \geq q_{j} y_{i j k} \quad \forall(i, j, k) \in A K .
\end{aligned}
$$

Formulation $H V R P_{6}$ has $|A K|+2 \sum_{i \in N}\left|K_{i}\right| 0-1$ and $|A K|$ continuous variables and $n+|K|+2|A K|+3 \sum_{i \in N}\left|K_{i}\right|$ linear constraints.

If $|K| \leq n$, both $H V R P_{5}$ and $H V R P_{6}$ have $O\left(n^{2}|K|\right)$ variables. But $H V R P_{5}$ has $O\left(n^{2}\right)$ constraints whereas formulation $H V R P_{6}$ has $O\left(n^{2}|K|\right)$ constraints.

\subsection{LP Relaxations}

Let $L P_{5}$ and $L P_{6}$ denote the LP bounds of $H V R P_{5}$ and $H V R P_{6}$, respectively. First we compare $L P_{5}$ and $L P_{6}$.

Theorem 3. $L P_{6} \geq L P_{5}$.

Proof. For $(a, b, y, g)$ feasible for the LP relaxation of $H V R P_{6},(a, b, y, f)$ where $f_{i j}=\sum_{k \in K_{i j}} g_{i j k}$ for all $(i, j) \in A$ is feasible for the LP relaxation of $H V R P_{5}$ and the two solutions have the same objective function value.

At this point, it is interesting to compare $L P_{5}$ and $L P_{6}$ with the LP bounds of formulations based on MTZ constraints. As these formulations are defined on different spaces, we project them onto the space of $a, b$ and $y$ to do the comparison.

Padberg and Sung [25] characterize the projection of the feasible set of the LP relaxation of MTZ formulation on the space of arc variables for the TSP. They show that the extreme rays correspond to directed cycles.

We first investigate the defining inequalities of the projection for the strongest MTZ formulation, i.e., $\mathrm{HVRP} \mathrm{P}_{4}$.

Lemma 1. The projection of the feasible set of the $L P$ relaxation of $\mathrm{HVRP}_{4}$ on the space of $a, b$ and $y$ is defined by constraints (10), (14)-(16), $0 \leq a_{i k} \leq 1,0 \leq b_{i k} \leq 1$ for all $i \in N$ and $k \in K_{i}, 0 \leq y_{i j k} \leq 1$ for all $(i, j, k) \in A K$, inequalities

$$
\sum_{(i, j) \in A_{C}} Q_{k} y_{i j k} \leq \sum_{i \in N_{C}}\left(Q_{k}-q_{i}\right)\left(a_{i k}+b_{i k}\right)
$$

for all $k \in K, N_{C} \subseteq\left\{i \in N: k \in K_{i}\right\}$ and $A_{C} \subseteq\{(i, j) \in A:(i, j, k) \in A K, i \in$ $\left.N_{C}, j \in N_{C}\right\}$ where arcs of $A_{C}$ make a directed cycle on the nodes of $N_{C}$, and inequalities

$$
\sum_{(i, j) \in A_{P}} Q_{k} y_{i j k}+\sum_{j:(j, s, k) \in A K} q_{j} y_{j s k} \leq \sum_{i \in N_{P}}\left(Q_{k}-q_{i}\right)\left(a_{i k}+b_{i k}\right)
$$


for all $k \in K, N_{P} \subseteq\left\{i \in N: k \in K_{i}\right\}$ and $A_{P} \subseteq\{(i, j) \in A:(i, j, k) \in A K, i \in$ $\left.N_{P}, j \in N_{P}\right\}$ where arcs of $A_{P}$ make a directed path on the nodes of $N_{P}$ which starts at node $s \in N_{P}$.

Proof. For $k \in K$, let $A_{k}=\{(i, j) \in A:(i, j, k) \in A K\}$ and $N_{k}=\left\{i \in N: k \in K_{i}\right\}$. Associate dual variable $\alpha_{i j}$ to constraint (20), $\sigma_{i}$ to constraint (21) and $\beta_{i}$ to constraint (22). Then by Farkas' lemma, for a given vector $y$, there exists $v$ satisfying (20)-(22) for $k$ if and only if

$$
\begin{aligned}
& \sum_{(i, j) \in A_{k}}\left(q_{j}\left(a_{j k}+b_{j k}\right)-Q_{k}\left(a_{i k}+b_{i k}-y_{i j k}\right)\right) \alpha_{i j} \\
& +\sum_{i \in N_{k}}\left(q_{i}\left(a_{i k}+b_{i k}\right)+\sum_{j:(j, i) \in A_{k}} q_{j} y_{j i k}\right) \sigma_{i} \leq \sum_{i \in N_{k}} Q_{k}\left(a_{i k}+b_{i k}\right) \beta_{i}
\end{aligned}
$$

for all $(\alpha, \sigma, \beta) \geq 0$ such that $-\sum_{j:(i, j) \in A_{k}} \alpha_{i j}+\sum_{j:(j, i) \in A_{k}} \alpha_{j i}+\sigma_{i} \leq \beta_{i}$ for all $i \in N_{k}$. Let $\phi$ be the set of $(\alpha, \sigma, \beta) \geq 0$ which satisfy this inequality. As set $\phi$ is a pointed polyhedral cone, it is sufficient to consider inequalities (54) for extreme rays of $\phi$. Next, we find a set of rays that includes the set of extreme rays of $\phi$. For $(\alpha, \sigma, \beta) \neq 0$ in $\phi$, let $A^{\prime}=\left\{(i, j) \in A_{k}: \alpha_{i j}>0\right\}$. If $A^{\prime}=\emptyset$ and if $(\alpha, \sigma, \beta)$ is an extreme ray, either $\beta_{i}>0$ for some $i \in N_{k}$ and all other entries are 0 or $\sigma_{i}=\beta_{i}>0$ for some $i \in N_{k}$ and all other entries are 0 . The corresponding inequalities (54) are $Q_{k}\left(a_{i k}+b_{i k}\right) \geq 0$ and $\left(Q_{k}-q_{i}\right)\left(a_{i k}+b_{i k}\right) \geq \sum_{j:(j, i, k) \in A K} q_{j} y_{j i k}$ for all $i \in N_{k}$. The first is implied by the nonnegativity of $a_{i k}$ and $b_{i k}$ and the latter is inequality (53) when $\left|N_{p}\right|=1$.

Now suppose that $A^{\prime} \neq \emptyset$. If there exists a directed cycle formed by arcs in $A_{C}$ in the graph with node set $N_{k}$ and arc set $A^{\prime}$, then for some small $\epsilon>0$, consider $\left(\alpha^{1}, \sigma, \beta\right)$ and $\left(\alpha^{2}, \sigma, \beta\right)$ where $\alpha_{i j}^{1}=\alpha_{i j}-\epsilon$ and $\alpha_{i j}^{2}=\alpha_{i j}+\epsilon$ for $(i, j) \in A_{C}$ and $\alpha_{i j}^{1}=\alpha_{i j}^{2}=\alpha_{i j}$ for $(i, j) \in A_{k} \backslash A_{C}$. Both $\left(\alpha^{1}, \sigma, \beta\right)$ and $\left(\alpha^{2}, \sigma, \beta\right)$ are in $\phi$ and $(\alpha, \sigma, \beta)=1 / 2\left(\alpha^{1}, \sigma, \beta\right)+1 / 2\left(\alpha^{2}, \sigma, \beta\right)$. So if $(\alpha, \sigma, \beta)$ is an extreme ray, then $\alpha_{i j}=0$ for all $(i, j) \in A_{k} \backslash A_{C}, \beta_{i}=\sigma_{i}=0$ for all $i \in N_{k}$ and $\alpha_{i j}=\alpha$ for all $(i, j) \in A_{C}$. Let $N_{C}$ be the nodes of the cycle. The corresponding inequality (54) is inequality (52).

Suppose that there is no directed cycle. Let $A_{P}$ be the arc set of a directed path in the graph with node set $N_{k}$ and arc set $A^{\prime}$ such that the first node of the path, say $s$ has no incoming arc and the last node, say $t$, has no outgoing arc. Note that by feasibility, $\beta_{t}>0$. For node $s$, either $\beta_{s}-\sigma_{s}>-\sum_{m:(s, m) \in A_{k}} \alpha_{s m}$ or $\sigma_{s}>0$. For some small $\epsilon>0$, define $\left(\alpha^{1}, \sigma^{1}, \beta^{1}\right)$ as $\alpha_{i j}^{1}=\alpha_{i j}-\epsilon$ for $(i, j) \in A_{P}$ and $\alpha_{i j}^{1}=\alpha_{i j}$ for $(i, j) \in A_{k} \backslash A_{P}, \beta_{t}^{1}=\beta_{t}-\epsilon$ and $\beta_{j}^{1}=\beta_{j}$ for $j \in N_{k} \backslash\{t\}, \sigma_{s}^{1}=\sigma_{s}-\epsilon$ if $\beta_{s}=\sigma_{s}-\sum_{m:(s, m) \in A_{k}} \alpha_{s m}$ and $\sigma_{s}^{1}=\sigma_{s}$ otherwise and $\sigma_{j}^{1}=\sigma_{j}$ for $j \in N_{k} \backslash\{s\}$. Also define $\left(\alpha^{2}, \sigma^{2}, \beta^{2}\right)$ as $\alpha_{i j}^{2}=\alpha_{i j}+\epsilon$ for $(i, j) \in A_{P}$ and $\alpha_{i j}^{2}=\alpha_{i j}$ for $(i, j) \in A_{k} \backslash A_{P}$, $\beta_{t}^{2}=\beta_{t}+\epsilon$ and $\beta_{j}^{2}=\beta_{j}$ for $j \in N_{k} \backslash\{t\}, \sigma_{s}^{2}=\sigma_{s}+\epsilon$ if $\beta_{s}=\sigma_{s}-\sum_{m:(s, m) \in A_{k}} \alpha_{s m}$ and $\sigma_{s}^{2}=\sigma_{s}$ otherwise and $\sigma_{j}^{2}=\sigma_{j}$ for $j \in N_{k} \backslash\{s\}$. As both $\left(\alpha^{1}, \sigma^{1}, \beta^{1}\right)$ and $\left(\alpha^{2}, \sigma^{2}, \beta^{2}\right)$ are in $\phi$ and $(\alpha, \sigma, \beta)=1 / 2\left(\alpha^{1}, \sigma^{1}, \beta^{1}\right)+1 / 2\left(\alpha^{2}, \sigma^{2}, \beta^{2}\right)$, if $(\alpha, \sigma, \beta)$ is extreme then $\alpha_{i j}=0$ for all $(i, j) \in A_{k} \backslash A_{P}, \beta_{j}=0$ for all $j \in N_{k} \backslash\{t\}, \sigma_{j}=0$ for all $j \in N_{k} \backslash\{s\}, \sigma_{s}=0$ if $0>\sigma_{s}-\sum_{m:(s, m) \in A_{k}} \alpha_{s m}$ and all positive entries have the same value. Let $N_{P}$ be the nodes of the path. If $\sigma_{s}=0$, then the corresponding inequality 
(54) is $\sum_{(i, j) \in A_{P}} Q_{j} y_{i j k} \leq \sum_{i \in N_{P} \backslash\{s\}}\left(Q_{k}-q_{i}\right)\left(a_{i k}+b_{i k}\right)+Q_{k}\left(a_{s k}+b_{s k}\right)$. This is dominated by the inequality (52) for $N_{C}=N_{P}$ and $A_{C}=A_{P} \cup\{(t, s)\}$. If $\sigma_{s}>0$, then the corresponding inequality (54) is inequality (53).

Different from the projection for the $T S P$, we end up with extreme rays related to both directed cycles and directed paths. But, if constraints (21) are replaced by the weaker constraints $v_{i k} \geq q_{i}\left(a_{i k}+b_{i k}\right)$ for all $i \in N$ and $k \in K_{i}$, then the resulting inequalities (53) are dominated by inequalities (52).

We can prove the following lemma in a similar way:

Lemma 2. The projection of the feasible set of the $\mathrm{LP}$ relaxation of $\mathrm{HVRP} \mathrm{P}_{3}$ on the space of $a, b$ and $y$ is defined by constraints (10), (14)-(16), $0 \leq a_{i k} \leq 1,0 \leq b_{i k} \leq 1$ for all $i \in N$ and $k \in K_{i}, 0 \leq y_{i j k} \leq 1$ for all $(i, j, k) \in A K$, inequalities

$$
\sum_{(i, j) \in A_{C}} \sum_{k \in K_{i j}} Q_{k} y_{i j k} \leq \sum_{i \in N_{C}} \sum_{k \in K_{i}} Q_{k}\left(a_{i k}+b_{i k}\right)-\sum_{i \in N_{C}} q_{i}
$$

for all $N_{C} \subseteq N$ and $A_{C} \subseteq\left\{(i, j) \in A: i \in N_{C}, j \in N_{C}\right\}$ where arcs of $A_{C}$ make a directed cycle on the nodes of $N_{C}$, and inequalities

$$
\sum_{(i, j) \in A_{P}} \sum_{k \in K_{i j}} Q_{k} y_{i j k}+\sum_{j:(j, s) \in A} \sum_{k \in K_{j s}} q_{j} y_{j s k} \leq \sum_{i \in N_{P}} \sum_{k \in K_{i}} Q_{k}\left(a_{i k}+b_{i k}\right)-\sum_{i \in N_{P}} q_{i}
$$

for all $N_{P} \subseteq N$ and $A_{P} \subseteq\left\{(i, j) \in A: i \in N_{P}, j \in N_{P}\right\}$ where arcs of $A_{P}$ make a directed path on the nodes of $N_{P}$ which starts at node $s \in N_{P}$.

We can also project out the $v_{i k}$ variables from the lifted formulation $H V R P_{4}$. The projection inequality is

$$
\begin{aligned}
& \sum_{(i, j) \in A_{k}}\left(q_{j}\left(a_{j k}+b_{j k}\right)-Q_{k}\left(a_{i k}+b_{i k}-y_{i j k}\right)+\left(Q_{k}-q_{i}-q_{j}\right) y_{j i k}\right) \alpha_{i j} \\
& \quad+\sum_{i \in N_{k}}\left(q_{i}\left(a_{i k}+b_{i k}\right)+\sum_{j:(j, i) \in A_{k}} q_{j} y_{j i k}\right) \sigma_{i} \\
& \leq \sum_{i \in N_{k}}\left(Q_{k}\left(a_{i k}+b_{i k}\right)-\sum_{j:(i, j, k) \in A K} q_{j} y_{i j k}-\left(Q_{k}-q_{i}-\max _{j:(i, j, k) \in A K} q_{j}\right) y_{0 i k}\right) \beta_{i}
\end{aligned}
$$

for $(\alpha, \sigma, \beta) \geq 0$ such that $-\sum_{j:(i, j) \in A_{k}} \alpha_{i j}+\sum_{j:(j, i) \in A_{k}} \alpha_{j i}+\sigma_{i} \leq \beta_{i}$ for all $i \in N_{k}$. So the projection is defined by constraints (10), (14)-(16), $0 \leq a_{i k} \leq 1,0 \leq b_{i k} \leq 1$ for all $i \in N$ and $k \in K_{i}, 0 \leq y_{i j k} \leq 1$ for all $(i, j, k) \in A K$, inequalities

$$
\sum_{(i, j) \in A_{C}}\left(Q_{k} y_{i j k}+\left(Q_{k}-q_{i}-q_{j}\right) y_{j i k}\right) \leq \sum_{i \in N_{C}}\left(Q_{k}-q_{i}\right)\left(a_{i k}+b_{i k}\right)
$$

for all $k \in K, N_{C} \subseteq\left\{i \in N: k \in K_{i}\right\}$ and $A_{C} \subseteq\{(i, j) \in A:(i, j, k) \in A K, i \in$ $\left.N_{C}, j \in N_{C}\right\}$ where arcs of $A_{C}$ make a directed cycle on the nodes of $N_{C}$, and

$$
\begin{aligned}
\sum_{(i, j) \in A_{P}}\left(Q_{k} y_{i j k}\right. & \left.+\left(Q_{k}-q_{i}-q_{j}\right) y_{j i k}\right)+\sum_{j:(j, s, k) \in A K} q_{j} y_{j s k}+\sum_{j:(t, j, k) \in A K} q_{j} y_{t j k} \\
& +\left(Q_{k}-q_{t}-\max _{j:(t, j, k) \in A K} q_{j}\right) y_{0 t k} \leq \sum_{i \in N_{P}}\left(Q_{k}-q_{i}\right)\left(a_{i k}+b_{i k}\right)
\end{aligned}
$$


for all $k \in K, N_{P} \subseteq\left\{i \in N: k \in K_{i}\right\}$ and $A_{P} \subseteq\{(i, j) \in A:(i, j, k) \in A K, i \in$ $\left.N_{P}, j \in N_{P}\right\}$ where arcs of $A_{P}$ make a directed path on the nodes of $N_{P}$ which starts at node $s \in N_{P}$ and ends at node $t \in N_{P}$.

We also need definitions of the projections of the feasible sets of the LP relaxations of $H V R P_{5}$ and $H V R P_{6}$ on the space of $a, b$ and $y$. Gouveia [13] finds the defining inequalities of this projection for the $C V R P$ with unit demands. He also gives the result for general demands without proof. The following two lemmas are modifications for the $H V R P$ and they can be proved in the same way as Result 1 in [13].

Lemma 3. The projection of the feasible set of the $L P$ relaxation of $H V R P_{6}$ on the space of $a, b$ and $y$ is defined by constraints (10), (14)-(16), $0 \leq a_{i k} \leq 1,0 \leq b_{i k} \leq 1$ for all $i \in N$ and $k \in K_{i}, 0 \leq y_{i j k} \leq 1$ for all $(i, j, k) \in A K$, and generalized large multistar inequalities (33) for all $k \in K$ and $S \subseteq\left\{i \in N: k \in K_{i}\right\}$.

Lemma 4. The projection of the feasible set of the $L P$ relaxation of $H V R P_{5}$ on the space of $a, b$ and $y$ is defined by constraints (10), (14)-(16), $0 \leq a_{i k} \leq 1,0 \leq b_{i k} \leq 1$ for all $i \in N$ and $k \in K_{i}, 0 \leq y_{i j k} \leq 1$ for all $(i, j, k) \in A K$, and generalized large multistar inequalities (32) for all $S \subseteq N$.

Theorem 4. $L P_{6} \geq L P_{4}$ and $L P_{5} \geq L P_{3}$.

Proof. The left hand side of inequality (33) is equal to

$$
\sum_{j \in S} Q_{k}\left(a_{j k}+b_{j k}\right)-\sum_{(i, j) \in(S: S): k \in K_{i j}} Q_{k} y_{i j k}-\sum_{(i, j) \in\left(N_{0} \backslash S: S\right): k \in K_{i j}} q_{i} y_{i j k} .
$$

Substituting this, we rewrite inequality (33) as

$$
\begin{aligned}
\sum_{j \in S}\left(Q_{k}-q_{j}\right)\left(a_{j k}+b_{j k}\right) \geq & \sum_{(i, j) \in(S: S): k \in K_{i j}} Q_{k} y_{i j k} \\
& +\sum_{(i, j) \in(S: N \backslash S): k \in K_{i j}} q_{j} y_{i j k}+\sum_{(i, j) \in\left(N_{0} \backslash S: S\right): k \in K_{i j}} q_{i} y_{i j k} .
\end{aligned}
$$

Now observe that inequality (33) dominates inequality (52) for $N_{C}=S$ and inequality (53) for $N_{P}=S$ for any $s \in S$. This together with Lemmas 1 and 3 shows that $L P_{6} \geq L P_{4}$. We can similarly prove that $L P_{5} \geq L P_{3}$.

We cannot compare $L P_{4}$ and $L P_{5}$ since there is no domination between inequalities (52) and (53) and inequalities (32).

Note also that although inequalities (33) dominate (55), in general there is no domination between (33) and (56). So we cannot compare $H V R P_{6}$ and $H V R P_{4}$ after lifting.

\subsection{Valid Inequalities}

Clearly, all valid inequalities for $H V R P_{4}$ discussed previously are also valid for $H V R P_{5}$ and $H V R P_{6}$. But projection results show that some of these inequalities are implied 
by formulations $H V R P_{5}$ and $H V R P_{6}$. Precisely, inequalities (32) are implied by constraints of the LP relaxation of $H V R P_{5}$. Also, as inequality (23) is the same as inequality (32) for $S=N$, it is implied. As a result of this, we expect the LP bound of $H V R P_{5}$ to be a better estimate of the optimal value compared to the LP bounds of MTZ formulations, since the case discussed in Proposition 1 cannot occur here.

Lemma 1 shows that inequalities (33) are implied by constraints of the LP relaxation of $\mathrm{HVRP}_{6}$. Again, as inequalities (25) are the same as inequalities (33) for $S=N$, they are implied by constraints of the LP relaxation of $H V R P_{6}$.

\section{Computational Results}

We conduct two experiments. The first one is to compare the lower bounds obtained from different formulations and to see if the valid inequalities and the lifting results presented in this paper improve these bounds.

Instances given in [12] are commonly used to test heuristic approaches. Here we use the 20 node problems for this first test. There are four problems instances: 3, 4, 5, and 6. Demands and coordinates of demand nodes are the same in all instances. Coordinates of the origin node is changed for instances 5 and 6 . There are 5 types of vehicles in instances 3 and 5, and 3 types of vehicles in instances 4 and 6 .

First, we solve the LP relaxations of the formulations without any valid inequalities and lifting. In Table 1, we report the results of this test. For each problem, we give the best upper bound (UB) as given in Gendreau et al. [10], Renaud and Boctor [27], and Taillard [30], and then for each formulation, the percentage gap $\left(\frac{U B-L B}{U B} 100\right.$ where LB is the lower bound). We do not report the solution times as they are small.

Notice that the lower bounds of the first four formulations are all the same and very poor due to the case discussed in Proposition 1. Lower bounds of flow formulations are much better.

Next, we discuss which inequalities we use in each formulation and report their effects. Inequalities (24) are valid for the six formulations and they are not implied in general. We choose $Q$ to be $Q_{1}, Q_{2}, \ldots, Q_{|K|}$ and also their greatest common divisor, $Q C$ and add the corresponding inequalities to all six formulations. When we take $Q$ to be $Q_{1}$, we obtain inequality $\sum_{i \in N} \sum_{k \in K_{i}} a_{i k} \geq\left\lceil\frac{q(N)}{Q_{1}}\right\rceil$. If the difference between $Q_{1}$ and $Q_{2}$ is big and if it is better not to use any vehicle of type 1 , then the right hand side of the above inequality may give a very poor lower bound. But then for $Q=Q_{2}$, we have the inequality $\sum_{i \in N} \sum_{k \in K_{i} \backslash\{1\}} a_{i k}+\sum_{i \in N}\left\lceil\frac{Q_{1}}{Q_{2}}\right\rceil a_{i 1} \geq\left\lceil\frac{q(N)}{Q_{2}}\right\rceil$ which may be more useful in case $a_{i 1}=0$ for all $i \in N$. This is the reasoning behind choosing $Q$ to be $Q_{1}, Q_{2}, \ldots, Q_{|K|}$. The reason for choosing $Q$ to be $Q C$ is that the correspond-

Table 1. Initial percentage gaps

\begin{tabular}{|c|c|c|c|c|c|c|c|}
\hline No. & $\mathrm{UB}$ & $H V R P_{1}$ & $H V R P_{2}$ & $H V R P_{3}$ & $H V R P_{4}$ & $H V R P_{5}$ & $H V R P_{6}$ \\
\hline 3 & 961.03 & 76.77 & 76.77 & 76.77 & 76.77 & 10.01 & 5.28 \\
4 & 6437.33 & 96.56 & 96.56 & 96.56 & 96.56 & 3.25 & 2.46 \\
5 & 1007.05 & 77.84 & 77.84 & 77.84 & 77.84 & 10.97 & 6.53 \\
6 & 6516.47 & 96.60 & 96.60 & 96.60 & 96.60 & 4.20 & 2.41 \\
\hline
\end{tabular}


Table 2. Percentage gaps after adding inequalities (24)

\begin{tabular}{|c|c|c|c|c|c|c|}
\hline No. & $H V R P_{1}$ & $H V R P_{2}$ & $H V R P_{3}$ & $H V R P_{4}$ & $H V R P_{5}$ & $H V R P_{6}$ \\
\hline 3 & 11.76 & 11.76 & 10.99 & 10.89 & 8.92 & 4.50 \\
4 & 1.91 & 1.91 & 1.91 & 1.91 & 1.67 & 0.90 \\
5 & 16.41 & 16.41 & 16.37 & 16.37 & 9.94 & 5.71 \\
6 & 3.45 & 3.45 & 3.44 & 3.44 & 2.64 & 0.86 \\
\hline
\end{tabular}

ing inequality (24) dominates inequality (23). Note that if $Q_{k}$ divides $q(N)$, then the corresponding inequality (24) is dominated by (24) for $Q=Q C$.

The results with these inequalities are reported in Table 2 . We observe a big decrease in gaps, especially for MTZ formulations. First two formulations still give the same lower bounds. Formulations $H V R P_{3}$ and $H V R P_{4}$ have better bounds than formulations $H V R P_{1}$ and $H V R P_{2}$ for instances 3, 5 and 6. Still, flow formulations have much better gaps. Adding inequalities (24) also improved the gaps for these formulations.

Instances 3 and 5 have larger gaps. These are the instances with 5 vehicle types. These results suggest that the number of vehicle types is an important factor in the difficulty of a problem.

Next, we add inequalities (25). These are undefined for $H V R P_{1}$ and are already implied by $\mathrm{HVRP}_{6}$. In Table 3, we report the percentage gaps obtained from the other four formulations with inequalities (24) and (25). We observe that there is more improvement for the hard instances, i.e., instances 3 and 5. Also the difference between formulations $\mathrm{HVRP} \mathrm{P}_{2}$ and $\mathrm{HVRP} \mathrm{P}_{3}$ is more apparent.

We use subtour elimination inequalities for sets $S$ of cardinality 2 and 3 . We use inequalities (26) and (27) for subsets of size 2 and inequalities (26) and (29) for subsets of size 3. In Table 4, we report the result with these inequalities as well as the previous inequalities. We see that adding these inequalities also improve the gaps in all formulations.

We use inequalities (34) and (35) defined by subsets $S$ of size 2 and 3. The results with these inequalities as well as the previous inequalities are given in Table 5. We do not

Table 3. Percentage gaps after adding inequalities (25)

\begin{tabular}{|c|c|c|c|c|}
\hline No. & $H V R P_{2}$ & $H V R P_{3}$ & $H V R P_{4}$ & $H V R P_{5}$ \\
\hline 3 & 11.47 & 8.93 & 8.36 & 6.79 \\
4 & 1.91 & 1.74 & 1.74 & 0.90 \\
5 & 16.41 & 15.70 & 15.19 & 7.50 \\
6 & 3.45 & 3.27 & 3.27 & 1.15 \\
\hline
\end{tabular}

Table 4. Percentage gaps after adding subtour elimination inequalities (26) for subsets of size 2 and 3 in $H V R P_{1}$ and $H V R P_{2}$, and (27) for subsets of size 2 and (29) for subsets of size 3 in $H V R P_{3}, H V R P_{4}$, $H V R P_{5}$, and $H V R P_{6}$

\begin{tabular}{|c|c|c|c|c|c|c|}
\hline No. & $H V R P_{1}$ & $H V R P_{2}$ & $H V R P_{3}$ & $H V R P_{4}$ & $H V R P_{5}$ & $H V R P_{6}$ \\
\hline 3 & 9.86 & 9.29 & 6.04 & 5.97 & 4.63 & 3.12 \\
4 & 1.58 & 1.58 & 1.23 & 1.23 & 0.77 & 0.77 \\
5 & 15.21 & 15.21 & 12.62 & 12.61 & 6.64 & 4.90 \\
6 & 3.21 & 3.21 & 2.83 & 2.83 & 0.97 & 0.76 \\
\hline
\end{tabular}


Table 5. Percentage gaps after adding inequalities (34) in $H V R P_{1}$ and $H V R P_{2}$ and (35) in $H V R P_{3}$ and $\mathrm{HVRP}_{4}$ for subsets of size 2 and 3

\begin{tabular}{|c|c|c|c|c|}
\hline No. & $H V R P_{1}$ & $H V R P_{2}$ & $H V R P_{3}$ & $H V R P_{4}$ \\
\hline 3 & 9.86 & 9.29 & 5.60 & 5.53 \\
4 & 1.58 & 1.58 & 1.11 & 1.11 \\
5 & 15.21 & 15.21 & 12.06 & 12.03 \\
6 & 3.21 & 3.21 & 2.07 & 2.07 \\
\hline
\end{tabular}

Table 6. Percentage gaps after lifting

\begin{tabular}{|c|c|c|c|c|}
\hline No. & $H V R P_{1}$ & $H V R P_{2}$ & $H V R P_{3}$ & $H V R P_{4}$ \\
\hline 3 & 9.86 & 8.66 & 5.59 & 5.06 \\
4 & 1.58 & 1.41 & 1.11 & 1.11 \\
5 & 15.21 & 15.21 & 11.99 & 11.10 \\
6 & 3.21 & 3.03 & 2.07 & 2.07 \\
\hline
\end{tabular}

use these inequalities with formulations $H V R P_{5}$ and $H V R P_{6}$ since inequalities (32) are implied by these formulations and the improvement was insignificant. Also, there is no improvement with formulations $H V R P_{1}$ and $H V R P_{2}$.

In Table 6, we present results with formulations strengthened with lifting. We observe that there is some improvement for the hard instances and disaggregated formulations.

To summarize, in Table 7, we present the percentage improvement in the gap due to a specific family of inequalities for each formulation and each instance. First we use only inequalities (24) and report the improvement with respect to the initial formulations. Then we also add inequalities (25) and compute the improvement with respect to the formulations with inequalities (24). We continue in this manner. For each formulation and family of inequalities, we also report the average improvement (in rows Ave). Inequalities (34) are not in the table as they did not improve the gaps.

We observe that valid inequalities are more useful in disaggregated formulations. Clearly, it is hard to derive strong inequalities without knowing the type of vehicle traversing an arc. Indeed, subtour elimination and generalized large multistar inequalities for the first two formulations are not very strong since the coefficients of arcs are computed as if they were traversed by the vehicle with the largest capacity.

We should note that, in the above results, subtour elimination inequalities and generalized multistar inequalities are included only for sets $S$ of cardinality 2 and 3 . We do not know whether these inequalities for larger subsets are useful in improving the LP bounds. To learn the answer, it is necessary to implement a cutting plane algorithm where these inequalities are separated. This is indeed an interesting further research question.

In the second experiment, we compute lower bounds for eight other instances from [12]. These are instances 13 to 20. In Table 8, for each instance, the number of demand nodes $n$, the number of vehicle types $|K|$, the best upper bound (UB), the lower bound given in [12] (LB), and the percentage gap between LB and UB are given.

We solve the LP relaxations of the six formulations for each instance. We use the valid inequalities and lifting results presented in the previous sections. We excluded the generalized large multistar inequalities to keep the size of the formulations tractable. The computation is carried out on a Sun Ultra $12 \times 400 \mathrm{MHz}$ using CPLEX 9 . 
Table 7. Percentage improvements in the gaps due to each family of valid inequalities and lifting

\begin{tabular}{|c|c|c|c|c|c|c|c|}
\hline Inequality & No. & $H V R P_{1}$ & $H V R P_{2}$ & $H V R P_{3}$ & $H V R P_{4}$ & $H V R P_{5}$ & $H V R P_{6}$ \\
\hline \multirow{5}{*}{ (24) } & 3 & 84.68 & 84.68 & 85.69 & 85.81 & 10.90 & 14.80 \\
\hline & 4 & 98.02 & 98.02 & 98.02 & 98.02 & 48.64 & 63.59 \\
\hline & 5 & 78.92 & 78.92 & 78.96 & 78.96 & 9.32 & 12.47 \\
\hline & 6 & 96.43 & 96.43 & 96.44 & 96.44 & 37.15 & 64.46 \\
\hline & Ave & 89.51 & 89.51 & 89.78 & 89.81 & 26.50 & 38.83 \\
\hline \multirow{5}{*}{ (25) } & 3 & - & 2.48 & 18.78 & 23.20 & 23.82 & - \\
\hline & 4 & - & 0.00 & 9.18 & 9.18 & 46.38 & - \\
\hline & 5 & - & 0.00 & 4.15 & 7.23 & 24.58 & - \\
\hline & 6 & - & 0.00 & 4.83 & 4.83 & 56.60 & - \\
\hline & Ave & & 0.62 & 9.24 & 11.11 & 37.85 & \\
\hline \multirow{5}{*}{ (26) } & 3 & 16.15 & 19.04 & - & - & - & - \\
\hline & 4 & 17.29 & 17.29 & - & - & - & - \\
\hline & 5 & 7.29 & 7.29 & - & - & - & - \\
\hline & 6 & 6.83 & 6.83 & - & - & - & - \\
\hline & Ave & 11.89 & 12.61 & - & - & - & - \\
\hline \multirow{5}{*}{$\begin{array}{l}\text { (27) } \\
\text { and } \\
(29)\end{array}$} & 3 & - & - & 32.33 & 28.58 & 31.77 & 30.58 \\
\hline & 4 & - & - & 28.88 & 28.88 & 13.54 & 13.54 \\
\hline & 5 & - & - & 19.61 & 16.98 & 11.42 & 14.15 \\
\hline & 6 & - & - & 13.51 & 13.51 & 15.60 & 11.02 \\
\hline & Ave & - & - & 23.58 & 21.99 & 18.08 & 17.32 \\
\hline \multirow{5}{*}{ (35) } & 3 & - & - & 7.26 & 7.36 & - & - \\
\hline & 4 & - & - & 9.92 & 9.92 & - & - \\
\hline & 5 & - & - & 4.39 & 4.62 & - & - \\
\hline & 6 & - & - & 27.00 & 27.00 & - & - \\
\hline & Ave & - & - & 12.14 & 12.23 & & \\
\hline \multirow{5}{*}{ Lifting } & 3 & 0.03 & 6.77 & 0.28 & 8.53 & - & - \\
\hline & 4 & 0.00 & 10.95 & 0.00 & 0.00 & - & - \\
\hline & 5 & 0.00 & 0.03 & 0.65 & 7.76 & - & - \\
\hline & 6 & 0.00 & 5.60 & 0.00 & 0.01 & - & - \\
\hline & Ave & 0.01 & 5.84 & 0.23 & 4.08 & & \\
\hline
\end{tabular}

Table 8. Characteristics, bounds, and percentage gaps for larger instances

\begin{tabular}{|c|c|c|c|c|c|}
\hline No. & $\mathrm{n}$ & $|K|$ & UB & LB in [12] & \% gap \\
\hline 13 & 50 & 6 & 2408.41 & 2119 & 12.02 \\
14 & 50 & 3 & 9119.03 & 8874 & 2.69 \\
15 & 50 & 3 & 2586.37 & 2264 & 12.46 \\
16 & 50 & 3 & 2741.5 & 2504 & 8.66 \\
17 & 75 & 4 & 1747.24 & 1380 & 21.02 \\
18 & 75 & 6 & 2373.63 & 2002 & 15.66 \\
19 & 100 & 3 & 8661.81 & 8290 & 4.29 \\
20 & 100 & 3 & 4047.55 & 3586 & 11.40 \\
\hline
\end{tabular}

The results are presented in Tables 9 and 10. For each instance and formulation, in Table 9, we give the percentage gap and in Table 10, the cpu time (in seconds) taken to solve the LP relaxation. We also give the averages over eight problems (in rows Ave).

We observe that the difference between formulations $H V R P_{1}$ and $H V R P_{2}$ is negligible for the gap. Solution time is about $8 \%$ more in the average for $H V R P_{2}$.

Also, the difference between the gaps of formulations $\mathrm{HVRP}_{3}$ and $\mathrm{HVRP}_{4}$ is very small whereas the average computation time for $H V R P_{4}$ is about 10 times the average 
Table 9. Percentage gaps for larger instances

\begin{tabular}{|c|c|c|c|c|c|c|}
\hline No. & $H V R P_{1}$ & $H V R P_{2}$ & $H V R P_{3}$ & $H V R P_{4}$ & $H V R P_{5}$ & $H V R P_{6}$ \\
\hline 13 & 8.00 & 7.99 & 6.67 & 6.00 & 5.01 & 1.77 \\
14 & 2.85 & 2.85 & 2.85 & 2.85 & 2.12 & 1.92 \\
15 & 11.33 & 11.33 & 10.65 & 10.56 & 7.63 & 3.20 \\
16 & 7.68 & 7.68 & 7.68 & 7.68 & 5.02 & 3.31 \\
17 & 9.29 & 9.29 & 9.29 & 9.29 & 5.87 & 3.28 \\
18 & 12.77 & 12.77 & 12.73 & 12.67 & 8.64 & 4.10 \\
19 & 3.44 & 3.44 & 3.44 & 3.44 & 1.41 & 1.01 \\
20 & 11.28 & 11.28 & 11.12 & 11.07 & 6.97 & 2.86 \\
\hline Ave & 8.33 & 8.33 & 8.05 & 7.95 & 5.33 & 2.68 \\
\hline
\end{tabular}

Table 10. Computation times for larger instances

\begin{tabular}{|c|c|c|c|c|c|c|}
\hline No. & $H V R P_{1}$ & $\mathrm{HVRP}_{2}$ & $\mathrm{HVRP}_{3}$ & $\mathrm{HVRP}_{4}$ & $H V R P_{5}$ & $H V R P_{6}$ \\
\hline 13 & 8.87 & 6.97 & 16.24 & 99.04 & 45.94 & 397.06 \\
\hline 14 & 5.42 & 3.55 & 6.90 & 79.71 & 39.75 & 175.64 \\
\hline 15 & 4.57 & 4.79 & 8.22 & 13.10 & 44.35 & 142.83 \\
\hline 16 & 5.85 & 4.33 & 5.42 & 20.51 & 29.06 & 142.11 \\
\hline 17 & 17.46 & 28.59 & 65.33 & 741.23 & 267.71 & 1344.81 \\
\hline 18 & 29.95 & 43.26 & 109.07 & 847.15 & 468.50 & 1922.80 \\
\hline 19 & 48.16 & 35.84 & 115.50 & 2589.63 & 1837.97 & 1721.18 \\
\hline 20 & 66.89 & 75.48 & 277.86 & 1628.40 & 2397.46 & 2904.04 \\
\hline Ave & 23.40 & 25.35 & 75.57 & 752.35 & 641.34 & 1093.81 \\
\hline
\end{tabular}

computation time for $\mathrm{HVRP}_{3}$. This implies that disaggregating the variable $u_{i}$ for each commodity is not likely to help in solving these problems.

Formulation $H V R P_{5}$ has better bounds and shorter computation times compared to formulation $\mathrm{HVRP} \mathrm{P}_{4}$ except for the last instance which has 100 nodes.

Formulation $\mathrm{HVRP}_{6}$ gives much better lower bounds compared to all other formulations. But the computation times are quite high. It may be impractical to use this formulation in a framework where many relaxations are to be solved.

The average computation time for the largest formulation $H V R P_{6}$ is about 46.7 times the time for the smallest formulation $H V R P_{1}$. The average percentage gap for formulation $H V R P_{1}$ is about 3.1 times the average percentage gap for formulation $H V R P_{6}$.

Finally, we observe that the improvement with respect to the lower bounds in the literature is considerable. The maximum gap was $21.02 \%$. With $H V R P_{6}$, the gap for the same instance is $3.28 \%$.

\section{Concluding Remarks}

In this paper, we formulated the $H V R P$ in different ways, compared the formulations and improved them using valid inequalities and lifting. The computational results showed that the lower bounds obtained from the strong formulations and the heuristic solutions in the literature are of good quality. These lower bounds also improved considerably upon the existing ones from [12]. 
Computational results also showed that strong formulations need a lot of computation time and smaller formulations give considerably worse lower bounds. A branch and cut algorithm may be implemented to see better the trade-off between the strength and size of the formulations.

Because of the success of branch and cut algorithms in the literature for the $C V R P$ (see e.g. Achuthan et al. [1], Baldacci et al. [4], and Lysgaard et al. [22]) and the improvement of lower bounds due to valid inequalities and lifting results presented in this paper, we believe that branch and cut may be an effective approach to solve $H V R P$ instances.

Finally, it would be interesting to develop algorithms to solve formulations that use only the $y_{i j k}$ and $a_{i k}$ variables and that are based on subtour elimination constraints (see Section 3.2.2) and see the effectiveness of these algorithms in solving HVRP instances.

\section{References}

1. Achuthan, N.R., Caccetta, L., Hill, S.P.: An improved branch and cut algorithm for the capacitated vehicle routing problem. Transportation Science 37, 153-169 (2003)

2. Araque, J.R., Hall, L.A., Magnanti, T.L.: Capacitated trees capacitated routing and associated polyhedra. Discussion paper, Center for Operations Research and Econometrics, Catholic University of Louvain, Belgium, 1990

3. Balas, E.: Facets of the knapsack polytope. Mathematical Programming 8, 146-164 (1975)

4. Baldacci, R., Hadjiconstantinou, E., Mingozzi, A.: An exact algorithm for the capacitated vehicle routing problem based on a two-commodity network flow formulation. Operations Research 52, 723-738 (2004)

5. Christof, T.: PORTA - a POlyhedron Representation Transformation Algorithm. Version 1.3.2 available at http://www.iwr.uni-heidelberg.de/groups/comopt/software/PORTA/ 1999

6. Desrochers, M., Verhoog, T.W.: A new heuristic for the fleet size and mix vehicle routing problem. Computers \& Operations Research 18, 263-274 (1991)

7. Desrochers, M., Laporte, G.: Improvements and extensions to the Miller-Tucker-Zemlin subtour elimination constraints. Operations Research Letters 10, 27-36 (1991)

8. Fisher, M.: Vehicle routing. In: M.O. Ball, T.L. Magnanti, C.L. Monma and G.L. Nemhauser (eds) Handbooks in OR MS, Vol. 8, Elsevier Science, 1995

9. Gavish, B., Graves, S.C.: The traveling salesman problem and related problems. Working Paper 7905, Graduate School of Management, University of Rochester, Rochester, 1979

10. Gendreau, M., Laporte, G., Musaraganyi, C., Taillard, E.D.: A tabu search heuristic for the heterogeneous fleet vehicle routing problem. Computers \& Operations Research 26, 1153-1173 (1999)

11. Gheysens, F., Golden, B., Assad, A.: A new heuristic for determining fleet size and composition. Mathematical Programming Study 26, 233-236 (1986)

12. Golden, B., Assad, A., Levy, L., Gheysens, F.: The fleet size and mix vehicle routing problem. Computers \& Operations Research 11, 49-66 (1984)

13. Gouveia, L.: A result on projection for the vehicle routing problem. European Journal of Operational Research 85, 610-624 (1995)

14. Gouveia, L., Pires, J.M.: The asymmetric traveling salesman problem and a reformulation of the MillerTucker-Zemlin constraints. European Journal of Operational Research 112, 134-146 (1999)

15. Hammer, P.L., Johnson, E.L., Peled, U.N.: Facets of regular 0-1 polytopes. Mathematical Programming 8, 179-206 (1975)

16. Kara, I., Laporte, G., Bektas, T.: A note on the lifted Miller-Tucker-Zemlin subtour elimination constraints for the capacitated vehicle routing problem. European Journal of Operational Research $\mathbf{1 5 8}$, 793-795 (2004)

17. Langevin, A., Soumis, F., Desrosiers, J.: Classification of traveling salesman problem formulations. Operations Research Letters 9, 127-132 (1990)

18. Laporte, G.: The vehicle routing problem: An overview of exact and approximate algorithms. European Journal of Operational Research 59, 345-358 (1992)

19. Laporte, G., Osman, I.H.: Routing problems: A bibliography. Annals of Operations Research 61, 227-262 (1995)

20. Letchford, A.N., Salazar-Gonzalez, J.J.: Projection results for vehicle routing. To appear in Mathematical Programming 
21. Letchford, A.N., Eglese, R.W., Lysgaard, J.: Multistars partial multistars and the capacitated vehicle routing problem. Mathematical Programming 94, 21-40 (2002)

22. Lysgaard, J., Letchford, A.N., Eglese, R.W.: A new branch and cut algorithm for the capacitated vehicle routing problem. Mathematical Programming 100, 423-445 (2004)

23. Mazur, D.R.: Integer programming approaches to a multi-facility location problem. Ph.D. Thesis, John Hopkins University, 1999

24. Miller, C., Tucker, A., Zemlin, R.: Integer programming formulations and traveling salesman problems. Journal of ACM 7, 326-329 (1960)

25. Padberg, M., Sung, T.Y.: An analytical comparison of different formulations of the traveling salesman problem. Mathematical Programming 52, 315-357 (1991)

26. Pochet, Y., Wolsey, L.A.: Integer knapsack and flow covers with divisible coefficients: Polyhedra optimization and separation. Discrete Applied Mathematics 59, 57-74 (1995)

27. Renaud, J., Boctor, F.F.: A sweep-based algorithm for the fleet size and mix vehicle routing problem. European Journal of Operational Research 140, 618-628 (2002)

28. Salhi, v., Sari, M., Saidi, D., Touati, N.A.C.: Adaptation of some vehicle fleet mix heuristics. OMEGA 20, 653-660 (1992)

29. Salhi, S., Rand, G.K.: Incorporating vehicle routing into the vehicle fleet composition problem. European Journal of Operational Research 66, 313-330 (1993)

30. Taillard, E.D.: A heuristic column generation method for the heterogeneous fleet VRP. RAIRO 33, 1-14 (1999)

31. Toth, P., Vigo, D.: Models relaxations and exact approaches for the capacitated vehicle routing problem. Discrete Applied Mathematics 123, 487-512 (2002)

32. Toth, P., Vigo, D.: The vehicle routing problem. SIAM monographs on discrete mathematics and applications. Philadelphia, 2002

33. Wassan, N.A., Osman, I.H.: Tabu search variants for the mix fleet vehicle routing problem. Journal of the Operational Research Society 53, 768-782 (2002)

34. Wolsey L.: Faces for a linear inequality in 0-1 variables. Mathematical Programming 8, 165-178 (1975)

35. Yaman, H.: The integer knapsack cover polyhedron. Working Paper 